\title{
CONTROLLABILITY PROBLEMS FOR THE 1-D WAVE EQUATION ON A HALF-AXIS WITH THE DIRICHLET BOUNDARY CONTROL
}

\author{
LARISSA V. FARDIGOLA ${ }^{1}$
}

\begin{abstract}
In this paper necessary and sufficient conditions of $\mathrm{L}^{\infty}$-controllability and approximate $\mathrm{L}^{\infty}$-controllability are obtained for the control system $w_{t t}=w_{x x}-q^{2} w, w(0, t)=u(t), x>0, t \in(0, T)$, where $q \geq 0, T>0, u \in \mathrm{L}^{\infty}(0, T)$ is a control. This system is considered in the Sobolev spaces.
\end{abstract}

Mathematics Subject Classification. 93B05, 35B37, 35L05.

Received September 2, 2010.

Published online September 19, 2011.

\section{INTRODUCTION}

Consider the wave equation on a half-axis

$$
w_{t t}=w_{x x}-q^{2} w, \quad x>0, t \in(0, T),
$$

controlled by the Dirichlet boundary condition

$$
w(0, t)=u(t), \quad t \in(0, T),
$$

where $q \geq 0$ is a given constant, $u \in L^{\infty}(0, T)$ is a control, $T>0$.

We should note that most of the papers investigating controllability of the wave equation deal with bounded domains and consider $L^{p}$-controllability $(2 \leq p \leq+\infty)[6,8-11,17]$ and many others. Controllability problems for distributed parameter systems on domains unbounded with respect to the space variables are investigated not enough. These problems for the wave equation on a half-plane in the context of controls bounded by a hard constant were investigated in [3]. Controllability of the wave equation in $\mathbb{R}^{3}$ were studied in [1]. Note that only $L^{\infty}$-controls can be realized practically. Controllability of the wave equation on a half-axis in the context of controls bounded by a hard constant were investigated in $[4,5,15,16]$. In $[15,16]$ the wave equation on a half-axis controlled by the Dirichlet boundary condition was studied for $q=0$. In $[4,5]$ the wave equation on a half-axis controlled by the Neumann boundary condition was studied for $q \geq 0$. In the present paper the most of the results of $[15,16]$ are extended to the case $q \geq 0$. Moreover, in the case $q \geq 0$ the results [4] on controllability of the wave equation (1.1) controlled by the Neumann boundary condition are also extended to the case of the Dirichlet boundary control here.

\footnotetext{
Keywords and phrases. Wave equation, half-axis, controllability problem, influence operator, Fourier transform, Sobolev space, Moore-Penrose inverse.

1 Mathematical Division, Institute for Low Temperature Physics and Engineering, 47 Lenin Ave., 61103 Kharkiv, Ukraine, fardigola@ukr.net
} 
In Section 3 we obtain necessary and sufficient conditions for $\mathrm{L}^{\infty}$-controllability and approximate $\mathrm{L}^{\infty}$ controllability of system (1.1), (1.2) at a given time $T>0$. The operator $\Psi_{T}, D\left(\Psi_{T}\right)=\left\{g \in H_{0}^{s}\right.$ | $g$ is odd and supp $g \subset[-T, T]\}$ describing the influence of a control on a target state is introduced and studied in the Sobolev spaces $H_{0}^{s}, s \leq 0$. It is proved that $\Psi_{T}$ is a bounded invertible operator, and $\Psi_{T}^{-1}$ is also bounded. This influence operator is similar to the one considered in [4], but it differs from it. The properties of the operator $\Psi_{T}$ obtained in Section 3 allows us to investigate the $\mathrm{L}^{\infty}$-controllability and the approximate $\mathrm{L}^{\infty}$-controllability problems at a given time. Controls solving these problems are found explicitly.

In the case $q=0$ necessary and sufficient conditions for approximate $\mathrm{L}^{\infty}$-controllability of control system (1.1), (1.2) were also established at a free time $T>0$ in [15]. Analogues result for the wave equation controlled by the Neumann boundary condition was obtained in [5] for $q=0$ too. Therein the time $T>0$ was not fixed, i.e. an appropriate time $T$ and a control $u$ had to be chose to solve approximate $\mathrm{L}^{\infty}$-controllability problem. In the case $q>0$ the question whether an initial state of control system (1.1), (1.2) was approximately $\mathrm{L}^{\infty}$-controllable at a free time was open. In Section 4 , to solve this problem, we consider an extension $\Psi$ of the operator $\Psi_{T}$. The domain of $\Psi$ contains functions with non-compact support. Unfortunately, this new influence operator $\Psi$ is not invertible. To solve the approximate $L^{\infty}$-controllability problem at a free time, we have to find an appropriate "inverse" operator for $\Psi$. We use the Moore-Penrose inverse (the generalized inverse) $\Psi^{+}$for the operator $\Psi[2,12,13]$. Application of the Moore-Penrose inverse operator is a key point of the present paper. Without loss of generality, we may consider the odd extension $\mathrm{W}$ of the solution $w$ to (1.1), (1.2) and consider system (3.2) instead of (1.1), (1.2) because these control systems are equivalent. Let $q>0, \mathrm{~W}^{0}=\left(\begin{array}{c}\mathrm{W}_{0}^{0} \\ \mathrm{~W}_{1}^{0}\end{array}\right) \in \widetilde{H}_{0}^{0} \times \widetilde{H}_{0}^{-1}$ be the initial state of control system (3.2), where $\widetilde{H}_{0}^{s}$ is the subspace of odd functions in $H_{0}^{s}, s \leq 0$. The state $\mathrm{W}^{0}$ is approximately $\mathrm{L}^{\infty}$-controllable iff $\mathrm{W}_{1}^{0}-\widehat{\Psi} \Psi^{+} \mathrm{W}_{0}^{0} \in \bar{\Psi}(N(\Psi))$, where the closure is considered in $\widetilde{H}_{0}^{-1}, N(\Psi)$ is the null space of $\Psi, \widehat{\Psi} g=\Psi \frac{\mathrm{d}}{\mathrm{d} t}(\operatorname{sgn} x g), g \in \widetilde{H}_{0}^{0}$, (Thm. 4.8). It is proved that the operator $\widehat{\Psi} \Psi^{+}$can be continued on the whole $\widetilde{H}_{0}^{0}$ (Lem. A.16), and $\widetilde{H}_{0}^{-1}$ is the closure of $\widehat{\Psi}(N(\Psi))$ with respect to the norm $\|\cdot\|_{0}^{-1}$ (Thm. 5.10). Thus, if $q>0$, then each state $\mathrm{W}^{0} \in \widetilde{H}_{0}^{0} \times \widetilde{H}_{0}^{-1}$ is approximately $\mathrm{L}^{\infty}$-controllable at a free time. It follows from [15] that if $q=0$, then the state $\mathrm{W}^{0} \in \widetilde{H}_{0}^{0} \times \widetilde{H}_{0}^{-1}$ is approximately $\mathrm{L}^{\infty}$-controllable at a free time iff $\mathrm{W}_{1}^{0}-\frac{\mathrm{d}}{\mathrm{d} x}\left(\operatorname{sgn} x \mathrm{~W}_{0}^{0}\right)=0$. We see that in the case $q>0$ the behavior of control system (1.1), (1.2) (and (3.2)) essentially differs from its behavior in the case $q=0$. This difference is generated by the properties of the influence operator $\Psi$. Indeed, $N(\Psi)=\{0\}$ and $\widehat{\Psi}(N(\Psi))=\{0\}$ if $q=0$, and $N(\Psi) \backslash\{0\} \neq \emptyset$ and $\overline{\Psi(N(\Psi))}=\widetilde{H}_{0}^{-1}$ if $q>0$. Here the closure is considered with respect to the norm $\|\cdot\|_{0}^{-1}$.

In Section 5 properties of $N(\Psi), \widehat{\Psi}(N(\Psi))$ and $N(\widehat{\Psi}), \Psi(N(\widehat{\Psi}))$ are studied. In fact, the main results of the present paper are based on the results of this section.

In Sections 3 and 6 the approximate $\mathrm{L}^{\infty}$-controllability problem is studied at a given time and a free time respectively when a control $u \in \mathrm{L}^{\infty}(0, T)$ is bounded by a given constant $U>0\left(\|u\|_{L^{\infty}(0, T)} \leq U\right)$.

In Section 7 the results of Sections 3-6 are illustrated by examples.

In the Appendix some properties of the operator $\Psi$ are proved.

\section{Notation}

Let us give definitions of the spaces used in the paper. Let $\mathcal{S}$ be the Schwartz space [14]

$$
\mathcal{S}=\left\{\varphi \in C^{\infty}(\mathbb{R}) \mid \forall m \in \mathbb{N} \forall l \in \mathbb{N} \sup \left\{\left|D^{m} \varphi(x)\right|\left(1+|x|^{2}\right)^{l} \mid x \in \mathbb{R}\right\}<+\infty\right\}
$$

and let $\mathcal{S}^{\prime}$ be the dual space, here $D=-i \partial / \partial x,|\cdot|$ is the Euclidean norm. 
Denote by $H_{l}^{s}(s, l \in \mathbb{R})$ the following Sobolev spaces:

$$
\begin{aligned}
H_{l}^{s} & =\left\{\varphi \in \mathcal{S}^{\prime} \mid\left(1+|D|^{2}\right)^{s / 2}\left(1+|x|^{2}\right)^{l / 2} \varphi \in L^{2}(\mathbb{R})\right\}, \\
\|\varphi\|_{l}^{s} & =\left(\int_{-\infty}^{\infty}\left|\left(1+|D|^{2}\right)^{s / 2}\left(1+|x|^{2}\right)^{l / 2} \varphi(x)\right|^{2} \mathrm{~d} x\right)^{1 / 2} .
\end{aligned}
$$

It is well known [7], Chapter 1, that $\|\varphi\|_{l}^{s} \leq\|\varphi\|_{l^{\prime}}^{s^{\prime}}, s \leq s^{\prime}, l \leq l^{\prime}, \varphi \in H_{l^{\prime}}^{s^{\prime}}$. Therefore, $H_{l}^{s} \supset H_{l^{\prime}}^{s^{\prime}}, s \leq s^{\prime}, l \leq l^{\prime}$.

Let $\mathcal{F}: \mathcal{S}^{\prime} \rightarrow \mathcal{S}^{\prime}$ be the Fourier transform operator. For $\varphi \in \mathcal{S}$ we have $(\mathcal{F} \varphi)(\sigma)=(2 \pi)^{-1 / 2} \int_{-\infty}^{\infty} \mathrm{e}^{-\mathrm{i} x \sigma} \varphi(x) \mathrm{d} x$ and $\langle\mathcal{F} f, \psi\rangle=\left\langle f, \mathcal{F}^{-1} \psi\right\rangle$ for $f \in \mathcal{S}^{\prime}, \psi \in \mathcal{S}$. It is well known [7], Chapter 1 , that $\mathcal{F} H_{0}^{s}=H_{s}^{0}$ and $\|\varphi\|_{0}^{s}=\|\mathcal{F} \varphi\|_{s}^{0}$, if $\varphi \in H_{0}^{s}$. A distribution $f \in \mathcal{S}^{\prime}$ is said to be $o d d$ if $\langle f, \varphi(\xi)\rangle=-\langle f, \varphi(-\xi)\rangle, \varphi \in \mathcal{S}$. We also use the spaces

$$
\widetilde{H}_{l}^{s}=\left\{\varphi \in H_{l}^{s} \mid \varphi \text { is odd }\right\}, \quad \widetilde{\mathbf{H}}=\widetilde{H}_{0}^{0} \times \widetilde{H}_{0}^{-1}
$$

with the norms $\|\cdot\|_{l}^{s},\|\varphi\|=\left(\left(\left\|\varphi_{0}\right\|_{0}^{0}\right)^{2}+\left(\left\|\varphi_{1}\right\|_{0}^{-1}\right)^{2}\right)^{1 / 2}$ respectively, $s, l \in \mathbb{R}$. Evidently, $\widetilde{H}_{l}^{s}$ is a (closed) subspace of $H_{l}^{s}, s, l \in \mathbb{R}$. Control system (1.1), (1.2) is considered in the spaces $H_{0}^{s}, s \leq 0$.

Further, for each function defined on a subset $Q$ of $\mathbb{R}$ we assume everywhere that it is extended on the whole $\mathbb{R}$ and vanish on $\mathbb{R} \backslash Q$. Throughout the paper the domain, the range and the null space (the kernel) of an operator $A$ are denoted by $D(A), R(A)$ and $N(A)$ respectively.

\section{Conditions for (approximate) Controllability at a given time}

Consider control system (1.1), (1.2) with the initial conditions

$$
\left\{\begin{array}{rl}
w(x, 0) & =\mathrm{W}_{0}^{0}(x) \\
w_{t}(x, 0) & =\mathrm{W}_{1}^{0}(x)
\end{array}, \quad x>0,\right.
$$

where $\mathrm{W}^{0}=\left(\begin{array}{c}\mathrm{W}_{0}^{0} \\ \mathrm{~W}_{1}^{0}\end{array}\right) \in \widetilde{\mathbf{H}}$. Let $\mathrm{W}(\cdot, t)$ be the odd extension of $\left(\begin{array}{c}w(\cdot, t) \\ w_{t}(\cdot, t)\end{array}\right)$. One can see that control problem (1.1), (1.2), (3.1) is equivalent to the following Cauchy problem

$$
\begin{gathered}
\frac{\mathrm{dW}}{\mathrm{d} t}=\left(\begin{array}{cc}
0 & 1 \\
\left(\left(\frac{\mathrm{d}}{\mathrm{d} x}\right)^{2}-q^{2}\right) & 0
\end{array}\right) \mathrm{W}-\left(\begin{array}{c}
0 \\
2 \delta^{\prime}(x)
\end{array}\right) u, \quad t \in(0, T), \\
\mathrm{W}(x, 0)=\mathrm{W}^{0}
\end{gathered}
$$

where $u \in \mathrm{L}^{\infty}(0, T)$ is a parameter (a control). Here $\delta$ is the Dirac distribution, $\delta=H^{\prime}, H$ is the Heaviside function: $H(\xi)=1$ if $\xi>0$, and $H(\xi)=0$ otherwise.

Let $\alpha>0, \Psi_{\alpha}: \mathcal{S}^{\prime} \rightarrow \mathcal{S}^{\prime}, D\left(\Psi_{\alpha}\right)=\left\{g \in \mathcal{S}^{\prime} \mid g\right.$ is odd and $\left.\operatorname{supp} g \subset[-\alpha, \alpha]\right\}$,

$$
\Psi_{\alpha} g=\mathcal{F}_{\sigma \rightarrow x}^{-1}\left(\frac{\sigma}{\sqrt{\sigma^{2}+q^{2}}}(\mathcal{F} g)\left(\sqrt{\sigma^{2}+q^{2}}\right)\right), \quad g \in D\left(\Psi_{\alpha}\right) .
$$

We have $\Psi_{\alpha} g=\frac{\mathrm{d}}{\mathrm{d} x} \mathcal{F}_{\sigma \rightarrow x}^{-1}\left(\frac{-\mathrm{i}}{\sqrt{\sigma^{2}+q^{2}}}(\mathcal{F} g)\left(\sqrt{\sigma^{2}+q^{2}}\right)\right)=\frac{\mathrm{d}}{\mathrm{d} x}(\Phi g), g \in D\left(\Psi_{\alpha}\right)$, where $\Phi$ was introduced and investigated in [4]. Evidently, if $q=0$, then $\Psi_{\alpha}=$ Id (where Id is the identity operator). 
Theorem 3.1. Let $\alpha>0$. Then the following assertions hold:

(i) $R\left(\Psi_{\alpha}\right)=D\left(\Psi_{\alpha}\right)$;

(ii) $R\left(\left.\Psi_{\alpha}\right|_{H_{0}^{s}}\right) \subset H_{0}^{s},\left.\Psi_{\alpha}\right|_{H_{0}^{s}}$ is bounded from $H_{0}^{s}$ to $H_{0}^{s}$ and $\left\|\left.\Psi_{\alpha}\right|_{H_{0}^{s}}\right\| \leq\left(1+q^{2}\right)^{-s / 2}, s \leq 0$;

(iii) $\Psi_{\alpha}$ is invertible, $D\left(\Psi_{\alpha}^{-1}\right)=R\left(\Psi_{\alpha}\right)=D\left(\Psi_{\alpha}\right)$;

(iv) $\left.\Psi_{\alpha}^{-1}\right|_{H_{0}^{s}}=\left(\left.\Psi_{\alpha}\right|_{H_{0}^{s}}\right)^{-1}$ and $\left.\Psi_{\alpha}^{-1}\right|_{H_{0}^{s}}$ is bounded from $H_{0}^{s}$ to $H_{0}^{s}, s \leq 0$;

(v) $\left(\Psi_{\alpha} g\right)=-\frac{\mathrm{d}}{\mathrm{d} x} \int_{|x|}^{\infty} J_{0}\left(q \sqrt{t^{2}-x^{2}}\right) g(t) \mathrm{d} t=g(x)-q x \int_{|x|}^{\infty} \frac{J_{1}\left(q \sqrt{t^{2}-x^{2}}\right)}{\sqrt{t^{2}-x^{2}}} g(t) \mathrm{d} t, g \in D\left(\Psi_{\alpha}\right) \cap H_{0}^{0}$;

(vi) $\left(\Psi_{\alpha}^{-1} f\right)=\mathcal{F}_{\mu \rightarrow t}^{-1}\left(\frac{\mu}{\sqrt{\mu^{2}-q^{2}}}(\mathcal{F} f)\left(\sqrt{\mu^{2}-q^{2}}\right)\right)=-\frac{\mathrm{d}}{\mathrm{d} t} \int_{|t|}^{\infty} I_{0}\left(q \sqrt{x^{2}-t^{2}}\right) f(x) \mathrm{d} x=$ $f(t)+q t \int_{|t|}^{\infty} \frac{I_{1}\left(q \sqrt{x^{2}-t^{2}}\right)}{\sqrt{x^{2}-t^{2}}} f(x) \mathrm{d} x, f \in D\left(\Psi_{\alpha}^{-1}\right) \cap H_{0}^{0} ;$

(vii) $\left\|\Psi_{\alpha} g\right\|_{L^{\infty}(-\alpha, \alpha)} \leq(1+q \alpha)\|g\|_{L^{\infty}(-\alpha, \alpha)}, g \in D\left(\Psi_{\alpha}\right) \cap L^{\infty}(-\alpha, \alpha)$;

(viii) $\left\|\Psi_{\alpha}^{-1} f\right\|_{L^{\infty}(-\alpha, \alpha)} \leq I_{0}(q \alpha)\|f\|_{L^{\infty}(-\alpha, \alpha)}, f \in D\left(\Psi_{\alpha}^{-1}\right) \cap L^{\infty}(-\alpha, \alpha)$;

(ix) $\left(\Psi_{\alpha}\left(\frac{\mathrm{d}}{\mathrm{d} t}\left(\operatorname{sgn} t \Psi_{\alpha}^{-1} f\right)\right)\right)(x)=(\operatorname{sgn} x f(x))^{\prime}+q \operatorname{sgn} x \int_{|x|}^{\infty} f(\xi) \frac{I_{1}(q(|x|-\xi))}{|x|-\xi} \mathrm{d} \xi=$ $(\operatorname{sgn} x f(x))^{\prime}+\frac{q}{2} \operatorname{sgn} x\left(f(x) *\left(I_{1}(q x) H(-x)\right)\right)(|x|), f \in D\left(\Psi_{\alpha}^{-1}\right)$.

Here $J_{\nu}(\xi)$ is the Bessel function, and $I_{\nu}(\xi)=i^{-\nu} J_{\nu}(\mathrm{i} \xi)$ is the modified Bessel function.

Assertions (i)-(vi) follow immediately from properties of $\Phi$ [4], Appendix. Assertions (vii), (viii) can be easily obtained by analogy with the corresponding properties of $\Phi$ [4], Appendix. Assertion (ix) is proved in Appendix of the present paper (Lem. A.15).

Taking into account [4], Proposition 3.2, Lemma 6.7, we conclude that the following theorem holds.

Theorem 3.2. Let $\mathrm{W}^{0} \in \mathcal{S}^{\prime} \times \mathcal{S}^{\prime}, u \in \mathrm{L}^{\infty}(0, T)$. Then

$$
\mathrm{W}(x, T)=E(x, T) *\left[\mathrm{~W}^{0}(x)-\left(\begin{array}{c}
\Psi_{T} \mathcal{U} \\
\Psi_{T} \frac{\mathrm{d}}{\mathrm{d} t}(\operatorname{sgn} t \mathcal{U})
\end{array}\right)(x)\right]
$$

where $\mathcal{U}(t)=u(t)(H(t)-H(t-T))-u(-t)(H(t+T)-H(t))$, W is the unique solution to $(3.2)-(3.3)$, * is the convolution with respect to $x$,

$$
E(x, t)=\frac{1}{2}\left(\begin{array}{cc}
\partial / \partial t & 1 \\
(\partial / \partial t)^{2} & \partial / \partial t
\end{array}\right)\left(J_{0}\left(q \sqrt{t^{2}-|x|^{2}}\right) \operatorname{sgn} t H\left(t^{2}-x^{2}\right)\right)
$$

In addition, $\left\|E(x, t) * f\left|\left\|\leq M_{q}^{t}\left|\|f \mid\|, M_{q}^{t}=\sqrt{\left(2 t^{2}+6\right)\left(1+q^{2}\right)}, t \in \mathbb{R}, f \in H_{0}^{0} \times H_{0}^{-1}\right.\right.\right.\right.$. Moreover, if $\mathrm{W}^{0} \in \widetilde{\mathbf{H}}$, then $\mathrm{W}(\cdot, t) \in \widetilde{\mathbf{H}}, t \in[0, T]$.

Let $U>0, T>0$. Denote $\mathcal{B}^{U}(0, T)=\left\{v \in L^{\infty}(0, T) \mid\|v\|_{L^{\infty}(0, T)} \leq U\right\}$. Obviously, $\bigcup_{U>0} \mathcal{B}^{U}(0, T)=$ $\mathrm{L}^{\infty}(0, T)$. For a given $T>0, \mathrm{~W}^{0} \in \widetilde{\mathbf{H}}$ denote by $\mathcal{R}_{T}^{U}\left(\mathrm{~W}^{0}\right)$ the set of the states $\mathrm{W}^{T} \in \widetilde{\mathbf{H}}$ for which there exists a control $u \in \mathcal{B}^{U}(0, T)$ such that problem $(3.2)-(3.3)$ has a unique solution $\mathrm{W}$ and $\mathrm{W}(\cdot, T)=\mathrm{W}^{T}$. Denote also $\mathcal{R}_{T}^{\infty}\left(\mathrm{W}^{0}\right)=\bigcup_{U>0} \mathcal{R}_{T}^{U}\left(\mathrm{~W}^{0}\right)$.

Definition 3.3. A state $\mathrm{W}^{0} \in \widetilde{\mathbf{H}}$ is called $\mathcal{B}^{U}$-controllable at a given time $T>0$ if 0 belongs to $\mathcal{R}_{T}^{U}\left(\mathrm{~W}^{0}\right)$ and approximately $\mathcal{B}^{U}$-controllable at a given time $T>0$ if 0 belongs to the closure of $\mathcal{R}_{T}^{U}\left(\mathrm{~W}^{0}\right)$ in $\widetilde{\mathbf{H}}$.

Definition 3.4. A state $\mathrm{W}^{0} \in \widetilde{\mathbf{H}}$ is called $\mathrm{L}^{\infty}$-controllable at a given time $T>0$ if 0 belongs to $\mathcal{R}_{T}^{\infty}\left(\mathrm{W}^{0}\right)$ and approximately $\mathrm{L}^{\infty}$-controllable at a given time $T>0$ if 0 belongs to the closure of $\mathcal{R}_{T}^{\infty}\left(\mathrm{W}^{0}\right)$ in $\widetilde{\mathbf{H}}$. 
With regard to Theorem 3.2 we have

$\mathcal{R}_{T}^{U}\left(\mathrm{~W}^{0}\right)=\left\{E(x, T) *\left[\mathrm{~W}^{0}(x)-\Psi_{T}\left(\begin{array}{c}\Psi_{T} \mathcal{U} \\ \Psi_{T} \frac{\mathrm{d}}{\mathrm{d} t}(\operatorname{sgn} t \mathcal{U})\end{array}\right)(x)\right] \mid \mathcal{U}\right.$ is odd, $\left.\|\mathcal{U}\|_{\mathrm{L}^{\infty}(\mathbb{R})} \leq U, \operatorname{supp} \mathcal{U} \in[-T, T]\right\}$.

The following theorem give us necessary and sufficient conditions for (approximate) $\mathcal{B}^{U}$-controllability.

Theorem 3.5. Let $\mathrm{W}^{0} \in \widetilde{\mathbf{H}}$ and a time $T>0$ be given. Then the following three assertions are equivalent

(i) $\mathrm{W}^{0}$ is $\mathcal{B}^{U}$-controllable at the time $T$;

(ii) $\mathrm{W}^{0}$ is approximately $\mathcal{B}^{U}$-controllable at the time $T$;

(iii) $\mathrm{W}^{0}$ satisfies the conditions

$$
\begin{gathered}
\operatorname{supp} \mathrm{W}_{0}^{0} \subset[-T, T] \\
\mathrm{W}_{1}^{0}-\Psi_{T} \frac{\mathrm{d}}{\mathrm{d} t}\left(\operatorname{sgn} t\left(\Psi_{T}^{-1} \mathrm{~W}_{0}^{0}\right)\right)=0 \\
\left\|\Psi_{T}^{-1} \mathrm{~W}_{0}^{0}\right\|_{\mathrm{L}^{\infty}(\mathbb{R})} \leq U .
\end{gathered}
$$

Moreover, the solution of the $\mathcal{B}^{U}$-controllability problem (the control $u$ ) is unique and

$$
u(t)=-\frac{\mathrm{d}}{\mathrm{d} t} \int_{t}^{T} I_{0}\left(q \sqrt{x^{2}-t^{2}}\right) \mathrm{W}_{0}^{0}(x) \mathrm{d} x=w_{0}^{0}(t)+q t \int_{t}^{T} \frac{I_{1}\left(q \sqrt{x^{2}-t^{2}}\right)}{\sqrt{x^{2}-t^{2}}} \mathrm{~W}_{0}^{0}(x) \mathrm{d} x \quad t \in(0, T) .
$$

Under conditions (3.7)-(3.8) $T_{*}=\max \operatorname{supp} \mathrm{W}_{0}^{0}$ is the optimal time and $u$ of the form (3.10) (for $\left.T=T_{*}\right)$ is the time-optimal control for the $\mathcal{B}^{U}$-controllability problem.

Proof. Let (3.7)-(3.8) hold. Put $\mathcal{U}=\Psi_{T}^{-1} \mathrm{~W}_{0}^{0}$. It follows from Theorem 3.1: (i), (iii), (viii) that $\mathcal{U}$ is odd, $\operatorname{supp} \mathcal{U} \subset[-T, T]$ and $\|\mathcal{U}\|_{L^{\infty}(\mathbb{R})} \leq U$. Denote by $u(t)$ its restriction on $[0, T]$. Then $\mathcal{U}(t)=u(t)(H(t)-H(t-$ $T))-u(-t)(H(t+T)-H(t))$. Using (3.9), we conclude that $u \in \mathcal{B}^{U}(0, T)$ and assertion (3.10) is true for it. Taking into account (3.8), we get

$$
\mathrm{W}_{1}^{0}=\Psi_{T} \frac{\mathrm{d}}{\mathrm{d} t}\left(\operatorname{sgn} t\left(\Psi_{T}^{-1} \mathrm{~W}_{0}^{0}\right)\right)=\Psi_{T} \frac{\mathrm{d}}{\mathrm{d} t}\left(\operatorname{sgn} t\left(\Psi_{T}^{-1} \Psi_{T} \mathcal{U}\right)\right)=\Psi_{T} \frac{\mathrm{d}}{\mathrm{d} t}(\operatorname{sgn} t \Omega \mathcal{U})
$$

Therefore, $(3.5)$ yields $\mathrm{W}(\cdot, T)=0$. Here $\mathrm{W}$ is the solution to (3.2), (3.3). Thus, $\mathrm{W}^{0}$ is $\mathcal{B}^{U}$-controllable at the time $T$. Therefore, it is approximately $\mathcal{B}^{U}$-controllable at this time. Put $T_{*}=\max \operatorname{supp} \mathrm{W}_{0}^{0}$. With regard to (3.5), we conclude that $T=T_{*}$ is the optimal time and $u$ of the form (3.10) (for $T=T_{*}$ ) is the time-optimal control for $\mathcal{B}^{U}$-controllability problem.

Let $\mathrm{W}_{0}^{0}$ be approximately $\mathcal{B}^{U}$-controllable at the time $T$. For each $n \in \mathbb{N}$ there exists a state $\mathrm{W}^{n} \in \mathcal{R}_{T}^{U}\left(\mathrm{~W}^{0}\right)$ such that $\left\|\mathrm{W}^{n}\right\| \mid<1 / n$. Using Theorem 3.2, we get

$$
\left\|\mathrm{W}_{0}^{0}-\Psi_{T} \mathcal{U}_{n}\right\|_{0}^{0} \rightarrow 0 \text { and }\left\|\mathrm{W}_{1}^{0}-\Psi_{T} \frac{\mathrm{d}}{\mathrm{d} t}\left(\operatorname{sgn} t \mathcal{U}_{n}\right)\right\|_{0}^{-1} \rightarrow 0 \quad \text { as } n \rightarrow \infty
$$

for some $u_{n} \in \mathcal{B}^{U}(0, T)$, where $\mathcal{U}_{n}(t)=u_{n}(t)(H(t)-H(t-T))-u_{n}(-t)(H(t+T)-H(t)), n \in \mathbb{N}$. According to Theorem 3.1: (i), (ii), we have supp $\mathrm{W}_{0}^{0} \subset[0, T]$ (i.e. (3.7) holds) and

$$
\left\|\Psi_{T}^{-1} \mathrm{~W}_{0}^{0}-\mathcal{U}_{n}\right\|_{0}^{0} \rightarrow 0 \quad \text { and } \quad\left\|\Psi_{T}^{-1} \mathrm{~W}_{1}^{0}-\left(\operatorname{sgn} t \mathcal{U}_{n}\right)^{\prime}\right\|_{0}^{-1} \rightarrow 0 \quad \text { as } n \rightarrow \infty .
$$

Put $\mathcal{U}=\Psi_{T}^{-1} \mathrm{~W}_{0}^{0}$. With regard to Theorem 3.1: (i), (iii), we get $\operatorname{supp} \mathcal{U} \subset[-T, T]$, $\mathcal{U}$ is odd. Since $u_{n} \in \mathcal{B}^{U}(0, T)$, we have $\|\mathcal{U}\|_{L^{\infty}(\mathbb{R})} \leq U$. According to Theorem 3.1: (ii), we obtain from here that (3.7), (3.9) are true. Taking 
into account (3.12), we obtain

$$
\left\|\mathcal{U}-\mathcal{U}_{n}\right\|_{0}^{0} \rightarrow 0 \quad \text { and } \quad\left\|(\operatorname{sgn} t \mathcal{U})^{\prime}-\left(\operatorname{sgn} t \mathcal{U}_{n}\right)^{\prime}\right\|_{0}^{-1} \rightarrow 0 \quad \text { as } n \rightarrow \infty .
$$

Hence, $(\operatorname{sgn} t \mathcal{U})^{\prime}=\Psi_{T}^{-1} \mathrm{~W}_{1}^{0}$ and (3.8) holds. The theorem is proved.

Remark 3.6. According to Theorem 3.1: (ix), condition (3.8) is equivalent to

$$
\mathrm{W}_{1}^{0}(x)=\left(\operatorname{sgn} x \mathrm{~W}_{0}^{0}(x)\right)^{\prime}+q \operatorname{sgn} x \int_{x}^{\infty} \mathrm{W}_{0}^{0}(\xi) \frac{I_{1}(q(x-\xi))}{x-\xi} \mathrm{d} \xi, \quad x>0 .
$$

Remark 3.7. Let $q=0$. Then $(3.8)$ is of the form $\mathrm{W}_{1}^{0}=\left(\operatorname{sgn} x \mathrm{~W}_{0}^{0}\right)^{\prime}$ and $(3.10)$ is of the form $u(t)=\mathrm{W}_{0}^{0}(t)$ on $[0, T]$. These conditions were obtained in [15] (for $q=0)$.

Remark 3.8. With regard to Theorem 3.1: (vii), (viii), condition

$$
\left\|\mathrm{W}_{0}^{0}\right\|_{\mathrm{L}^{\infty}(\mathbb{R})} \leq \frac{U}{I_{0}(q T)}
$$

is sufficient for (3.9) but it is not necessary for (3.9), according to Example 7.1, if $q>0$. Due to the same theorem, condition

$$
\left\|\mathrm{W}_{0}^{0}\right\|_{\mathrm{L}^{\infty}(\mathbb{R})} \leq U(1+q T)
$$

is necessary for (3.9) but it is not sufficient for (3.9), according to Example 7.1, if $q>0$. If $q=0$, then we obtain from here that (3.9), (3.13), and (3.14) are equivalent to $\left\|\mathrm{W}_{0}^{0}\right\|_{\mathrm{L}^{\infty}(\mathbb{R})} \leq U$.

Corollary 3.9. Let $\mathrm{W}^{0} \in \widetilde{\mathbf{H}}$ and a time $T>0$ be given. Then

(i) $\mathrm{W}^{0}$ is approximately $\mathrm{L}^{\infty}$-controllable at the time $T$ iff (3.7) and (3.8) hold;

(ii) $\mathrm{W}^{0}$ is $\mathrm{L}^{\infty}$-controllable at the time $T$ iff $\mathrm{W}_{0}^{0} \in \mathrm{L}^{\infty}(0, T)$ and $(3.7)$, (3.8) hold.

Proof. Let us prove (i). Reasoning as in the proof of Theorem 3.5, we conclude that (3.7) and (3.8) are necessary for approximate $\mathrm{L}^{\infty}$-controllability. To prove their sufficiency, we consider a sequence $\left\{\mathrm{W}_{0}^{n}\right\}_{n \in \mathbb{N}} \subset \mathrm{L}^{\infty}(\mathbb{R})$ such that $\operatorname{supp} \mathrm{W}_{0}^{n} \subset[-T, T], n \in \mathbb{N}$, and $\left\|\mathrm{W}_{0}^{0}-\mathrm{W}_{0}^{n}\right\|_{0}^{0} \rightarrow 0$ as $n \rightarrow \infty$. Put $\mathcal{U}=\Psi_{T}^{-1} \mathrm{~W}_{0}^{0}, \mathcal{U}_{n}=\Psi_{T}^{-1} \mathrm{~W}_{0}^{n}$, $n \in \mathbb{N}$. According to Theorem 3.1: (i), (ii), (iv), (viii), we have $\mathcal{U} \in L^{2}(\mathbb{R}), \mathcal{U}_{n} \in \mathrm{L}^{\infty}(\mathbb{R})$, $\operatorname{supp} \mathcal{U} \subset[-T, T]$, $\operatorname{supp} \mathcal{U}_{n} \subset[-T, T], n \in \mathbb{N}$, and $\left\|\mathcal{U}-\mathcal{U}_{n}\right\|_{0}^{0} \rightarrow 0$ as $n \rightarrow \infty$. With regard to Theorem 3.1: (ii) and (3.8), we get

$$
\left\|\mathrm{W}_{0}^{1}-\Psi_{T} \frac{\mathrm{d}}{\mathrm{d} t}\left(\operatorname{sgn} t \mathcal{U}_{n}\right)\right\|_{0}^{-1}=\left\|\Psi_{T} \frac{\mathrm{d}}{\mathrm{d} t}\left(\operatorname{sgn} t\left(\mathcal{U}-\mathcal{U}_{n}\right)\right)\right\|_{0}^{-1}=\left\|\mathcal{U}-\mathcal{U}_{n}\right\|_{0}^{0} \rightarrow 0 \quad \text { as } n \rightarrow 0 .
$$

Applying Theorem 3.2, we conclude that $\mathrm{W}_{0}^{0}$ is approximately $\mathrm{L}^{\infty}$-controllable at the time $T>0$.

Theorem 3.5 and Remark 3.8 yield assertion (ii). The corollary is proved.

Examples 7.2, 7.6 illustrate Theorem 3.5 and Corollary 3.9.

\section{Conditions for approximate $\mathrm{L}^{\infty}$-COntrollability at A Free time}

Consider control system (1.1), (1.2), (3.1) and equivalent system (3.2), (3.3), where $T>0$ is a parameter.

Definition 4.1. A state $\mathrm{W}^{0} \in \widetilde{\mathbf{H}}$ is called approximately $\mathrm{L}^{\infty}$-controllable (at a free time) if 0 belongs to the closure of $\bigcup_{T>0} \mathcal{R}_{T}^{\infty}\left(\mathrm{W}^{0}\right)$ in $\tilde{\mathbf{H}}$.

Definition 4.2. System $(3.2)$, (3.3) is called approximately $\mathrm{L}^{\infty}$-controllable if each state $\mathrm{W}^{0} \in \widetilde{\mathbf{H}}$ is approximately $\mathrm{L}^{\infty}$-controllable. 
Let us consider an extension of $\Psi_{\alpha}$ on a space of functions with non-compact support. Let $\Psi: H_{0}^{0} \rightarrow H_{0}^{0}$, $D(\Psi)=\widetilde{H}_{0}^{0}$,

$$
\Psi g=\mathcal{F}_{\sigma \rightarrow x}^{-1}\left(\frac{\sigma}{\sqrt{\sigma^{2}+q^{2}}}(\mathcal{F} g)\left(\sqrt{\sigma^{2}+q^{2}}\right)\right), \quad g \in D(\Psi) .
$$

Evidently, $\Psi g=\Psi_{\alpha} g, g \in D(\Psi) \cap D\left(\Psi_{\alpha}\right)$. One can see that if $q=0$, then $\Psi=\mathrm{Id}$.

Theorem 4.3. Let $q>0$. Then the following assertions hold:

(i) $R(\Psi)=\left\{f \in \widetilde{H}_{0}^{0} \mid \exists \tilde{f} \in H_{0}^{1 / 2} \mathcal{F} f=\sqrt{|\sigma|} \mathcal{F} \widetilde{f}\right\}, \overline{R(\Psi)}=\widetilde{H}_{0}^{0}$;

(ii) $\Psi$ is bounded and $\|\Psi\| \leq 1$;

(iii) $N(\Psi)=\left\{g \in \widetilde{H}_{0}^{0} \mid \operatorname{supp} \mathcal{F} g \subset[-q, q]\right\}$;

(iv) $(\Psi g)(x)=-\frac{\mathrm{d}}{\mathrm{d} x} \int_{|x|}^{\infty} J_{0}\left(q \sqrt{t^{2}-x^{2}}\right) g(t) \mathrm{d} t=g(x)-q x \int_{|x|}^{\infty} \frac{J_{1}\left(q \sqrt{t^{2}-x^{2}}\right)}{\sqrt{t^{2}-x^{2}}} g(t) \mathrm{d} t, g \in D(\Psi)$;

Proof. Lemma A.13 implies (i). By analogy with properties of $\Phi$ [4], Appendix, we obtain assertions (ii), (iv). With regard to the definition of $\Psi$, we conclude that (iii) is true. The theorem is proved.

Taking into account (3.5), consider an extension of $\Psi_{\alpha} \frac{\mathrm{d}}{\mathrm{d} t} \operatorname{sgn} t$ on a space of functions with non-compact support. Let $\widehat{\Psi}: H_{0}^{0} \rightarrow H_{0}^{-1}, D(\widehat{\Psi})=D(\Psi)=\widetilde{H}_{0}^{0}$,

$$
\widehat{\Psi} g=\frac{\mathrm{d}}{\mathrm{d} x} \mathcal{F}_{\sigma \rightarrow x}^{-1}\left((\mathcal{F}(\operatorname{sgn} t g))\left(\sqrt{\sigma^{2}+q^{2}}\right)\right), \quad g \in D(\widehat{\Psi}) .
$$

Evidently, $\widehat{\Psi} g=\Psi_{\alpha} \frac{\mathrm{d}}{\mathrm{d} t}(\operatorname{sgn} t g), g \in D(\widehat{\Psi}) \cap D\left(\Psi_{\alpha}\right)$. One can see that if $q=0$, then $\widehat{\Psi}=\frac{\mathrm{d}}{\mathrm{d} x} \operatorname{sgn} x$.

If $\mathrm{W}^{0} \in \widetilde{\mathbf{H}}, T>0, u \in \mathrm{L}^{\infty}(0, T)$, then formula (3.5) can be represented in the form

$$
\mathrm{W}(x, T)=E(x, T) *\left[\mathrm{~W}^{0}(x)-\left(\begin{array}{c}
\Psi \mathcal{U} \\
\widehat{\Psi} \mathcal{U}
\end{array}\right)(x)\right],
$$

where $\mathcal{U}(t)=u(t)(H(t)-H(t-T))-u(-t)(H(t+T)-H(t))$.

By analogy with Theorem 4.3 , we obtain

Theorem 4.4. Let $q>0$. Then the following assertions hold:

(i) $R(\widehat{\Psi})=\left\{f \in \widetilde{H}_{0}^{-1} \mid \exists \tilde{f} \in H_{0}^{-1 / 2} \mathcal{F} f=\sqrt{|\sigma|} \mathcal{F} \widetilde{f}\right\}, \overline{R(\widehat{\Psi})}=\widetilde{H}_{0}^{-1}$;

(ii) $\widehat{\Psi}$ is bounded and $\|\widehat{\Psi}\| \leq 1$;

(iii) $N(\widehat{\Psi})=\left\{g \in \widetilde{H}_{0}^{0} \mid \operatorname{supp} \mathcal{F}(\operatorname{sgn} t g) \subset[-q, q]\right\}$;

(iv) $(\widehat{\Psi} g)(x)=\frac{\mathrm{d}}{\mathrm{d} x}\left(\operatorname{sgn} x g(x)-q \int_{|x|}^{\infty} \frac{t J_{1}\left(q \sqrt{t^{2}-x^{2}}\right)}{\sqrt{t^{2}-x^{2}}} g(t) \mathrm{d} t\right), g \in D(\widehat{\Psi})$;

We have used the inverse operator for $\Psi_{\alpha}$ to solve controllability problem at a given time. But $\Psi$ is not invertible according to Theorem 4.3. Let us use the Moore-Penrose inverse for the operator $\Psi[2,12,13]$ to solve approximate controllability problem at a free time. Decompose the space $\widetilde{H}_{0}^{0}$ into a direct sum of subspaces. Denote $\mathbf{L}_{1}=(N(\Psi))^{\perp}, \mathbf{L}_{2}=N(\Psi)$. Evidently, $\mathbf{L}_{1}=\left\{g \in \widetilde{H}_{0}^{0} \mid \operatorname{supp} \mathcal{F} g \subset \mathbb{R} \backslash(-q, q)\right\}, \mathbf{L}_{2}=\left\{g \in \widetilde{H}_{0}^{0} \mid\right.$ $\operatorname{supp} \mathcal{F} g \subset[-q, q]\}$. We have $\widetilde{H}_{0}^{0}=\mathbf{L}_{1} \oplus \mathbf{L}_{2}$. According to Theorem 4.3, the restriction $\left.\Psi\right|_{\mathbf{L}_{1}}$ is an invertible operator. Denote $\Psi^{+}=\left(\left.\Psi\right|_{\mathbf{L}_{1}}\right)^{-1}, D\left(\Psi^{+}\right)=R(\Psi)$. Here $\Psi^{+}$is called the Moore-Penrose inverse for $\Psi$. We have $\Psi \Psi^{+}=\mathrm{Id}, \Psi^{+} \Psi=P_{\mathbf{L}_{1}}$, where $P_{\mathbf{L}_{j}}$ is the projector on $\mathbf{L}_{j}$ in $\widetilde{H}_{0}^{0}, j=1,2$. One can see that

$$
\Psi^{+} f=\mathcal{F}_{\mu \rightarrow t}^{-1}\left(\frac{\mu H\left(\mu^{2}-q^{2}\right)}{\sqrt{\mu^{2}-q^{2}}}(\mathcal{F} f)\left(\sqrt{\mu^{2}-q^{2}}\right)\right), \quad f \in D\left(\Psi^{+}\right) .
$$


Denote

$$
\begin{array}{ll}
\psi_{\infty}(t, x)=\frac{2}{\pi} \operatorname{sgn} x H\left(x^{2}-t^{2}\right) t \int_{|t|}^{\infty}\left(\frac{\sin (q v)}{v}\right)^{\prime} \frac{\mathrm{d} v}{\sqrt{v^{2}+x^{2}-t^{2}}}, & (t, x) \in \mathbb{R}^{2}, \\
\psi_{0}(t, x)=\frac{2}{\pi} \operatorname{sgn} x H\left(t^{2}-x^{2}\right) t \int_{\sqrt{t^{2}-x^{2}}}^{|t|}\left(\frac{\sin (q v)}{v}\right)^{\prime} \frac{\mathrm{d} v}{\sqrt{v^{2}+x^{2}-t^{2}}}, & (t, x) \in \mathbb{R}^{2} .
\end{array}
$$

Due to Lemma A.14, (4.3) and the definition of $\Psi^{+}$, we have

Theorem 4.5. Let $q>0$. Then the following assertions hold:

(i) $R\left(\Psi^{+}\right)=\mathbf{L}_{1}$;

(ii) $\Psi^{+}$is invertible, $\left(\Psi^{+}\right)^{-1}=\left.\Psi\right|_{\mathbf{L}_{1}}, D\left(\left(\Psi^{+}\right)\right)^{-1}=R\left(\Psi^{+}\right)=\mathbf{L}_{1}$;

(iii) $\left(\Psi^{+} f\right)(t)=f(t)-\int_{|t|}^{\infty} \psi_{\infty}(t, x) f(x) \mathrm{d} x+\int_{0}^{|t|} \psi_{0}(t, x) f(x) \mathrm{d} x, f \in R(\Psi)$;

The operator $\widehat{\Psi} \Psi^{+}=\Psi \frac{\mathrm{d}}{\mathrm{d} t} \operatorname{sgn} t \Psi^{+}$is a generalization of $\Psi_{\alpha} \frac{\mathrm{d}}{\mathrm{d} t} \operatorname{sgn} t \Psi_{\alpha}^{-1}$. To investigate approximate controllability at a free time, we study the operator $\widehat{\Psi} \Psi^{+}$because the operator $\Psi_{\alpha} \frac{\mathrm{d}}{\mathrm{d} t} \operatorname{sgn} t \Psi_{\alpha}^{-1}$ play an important role in the study of the controllability problem at a given time. Taking into account Lemma A.16, we can continue the operator $\widehat{\Psi} \Psi^{+}$on $\widetilde{H}_{0}^{0}$. Denote $\Upsilon: H_{0}^{0} \rightarrow H_{0}^{-1}, D(\Upsilon)=\widetilde{H}_{0}^{0}$,

$$
\Upsilon f=(\operatorname{sgn} x f)^{\prime}+q^{2} \operatorname{sgn} x \mathcal{F}_{\sigma \rightarrow x}^{-1}\left(\frac{\mathrm{i} \operatorname{sgn} \sigma(\mathcal{F} f)(\sigma)}{|\sigma|+\sqrt{\sigma^{2}+q^{2}}}\right)=\operatorname{sgn} x \mathcal{F}_{\sigma \rightarrow x}^{-1}\left(\operatorname{isgn} \sigma \sqrt{\sigma^{2}+q^{2}}(\mathcal{F} f)(\sigma)\right), \quad f \in D(\Upsilon) .
$$

According to Lemma A.16, $\Upsilon f=\widehat{\Psi} \Psi^{+} f, f \in R(\Psi)$. One can see that if $q=0$, then $\Upsilon=\frac{\mathrm{d}}{\mathrm{d} x} \operatorname{sgn} x$. By analogy with the modified Bessel function

$$
I_{p}(x)=\frac{x^{p}}{2^{p-1} \Gamma(1 / 2) \Gamma(p+1 / 2)} \int_{0}^{1}\left(1-\xi^{2}\right)^{p-1 / 2} \cosh (\xi x) \mathrm{d} \xi, \quad p \geq 0,
$$

we introduce the following cylindric function

$$
L_{p}(x)=\frac{x^{p}}{2^{p-1} \Gamma(1 / 2) \Gamma(p+1 / 2)} \int_{0}^{1}\left(1-\xi^{2}\right)^{p-1 / 2} \sinh (\xi x) \mathrm{d} \xi, \quad p \geq 0 .
$$

Using the definition of $\Upsilon$, Lemmas A.16, A.18, and Corollary A.19, we obtain

Theorem 4.6. Let $q>0$. Then the following assertions hold:

(i) $R(\Upsilon)=\widetilde{H}_{0}^{-1}$;

(ii) $\Upsilon$ is bounded and $\|\Upsilon\| \leq \sqrt{1+q^{2}}$;

(iii) $\Upsilon$ is invertible, $\Upsilon^{-1} g=\mathcal{F}_{\sigma \rightarrow x}^{-1}\left(\frac{\mathcal{F}(\operatorname{sgn} \xi g)(\sigma)}{i \operatorname{sgn} \sigma \sqrt{\sigma^{2}+q^{2}}}\right), g \in D\left(\Upsilon^{-1}\right)=R(\Upsilon)=\widetilde{H}_{0}^{-1}$;

(iv) $\Upsilon^{-1}$ is bounded, $\left\|\Upsilon^{-1}\right\| \leq \frac{\sqrt{1+q^{2}}}{q}$;

(v) $\Upsilon f=(\operatorname{sgn} x f(x))^{\prime}+\frac{q}{2} \operatorname{sgn} x\left(f(x) * \frac{I_{1}(q|x|)-L_{1}(q x)}{x}\right), f \in \widetilde{H}_{0}^{0}$.

Theorem 4.7. Let $q=0$. A state $\mathrm{W}^{0} \in \widetilde{\mathbf{H}}$ is approximately $\mathrm{L}^{\infty}$-controllable at a free time iff

$$
\mathrm{W}_{1}^{0}-\left(\operatorname{sgn} x \mathrm{~W}_{0}^{0}\right)^{\prime}=0
$$

We obtain this assertion from [15], Theorem 1.1. Note also that $\mathrm{W}_{1}^{0}-\left(\operatorname{sgn} x \mathrm{~W}_{0}^{0}\right)^{\prime}=\mathrm{W}_{1}^{0}-\Upsilon \mathrm{W}_{0}^{0}$ if $q=0$. 
Theorem 4.8. A state $\mathrm{W}^{0} \in \widetilde{\mathbf{H}}$ is approximately $\mathrm{L}^{\infty}$-controllable at a free time iff

$$
\mathrm{W}_{1}^{0}-\Upsilon \mathrm{W}_{0}^{0} \in \overline{\widehat{\Psi}(N(\Psi))},
$$

where the closure is considered with respect to the norm $\|\cdot\|_{0}^{-1}$.

Proof. Let $q=0$. Theorem 4.7 yields the assertion of this theorem because condition (4.7) is equivalent to condition (4.6) in this case. Now let $q>0$.

Necessity of (4.7). Let $\mathrm{W}^{0} \in \widetilde{\mathbf{H}}$ be approximately $\mathrm{L}^{\infty}$-controllable. Then for any $n \in \mathbb{N}$ there exist $T_{n}>0$ and $u_{n} \in \mathrm{L}^{\infty}\left(0, T_{n}\right)$ such that for the solution $\mathrm{W}_{n}$ to (3.2), (3.3) we have $\left\|\mathrm{W}_{n}\left(\cdot, T_{n}\right)\right\| \| \rightarrow 0$ as $n \rightarrow \infty$. Taking into account Lemma A.12 and $\left(3.5^{\prime}\right)$, we obtain $\left\|\mathrm{W}_{0}^{0}-\mathrm{W}_{0}^{n}\right\|_{0}^{0} \rightarrow 0$ and $\left\|\mathrm{W}_{1}^{0}-\mathrm{W}_{1}^{n}\right\|_{0}^{-1} \rightarrow 0$ as $n \rightarrow \infty$, where $\mathrm{W}_{0}^{n}=\Psi \mathcal{U}_{n}, \mathrm{~W}_{1}^{n}=\widehat{\Psi} \mathcal{U}_{n}, \mathcal{U}_{n}(t)=u_{n}(t)(H(t)-H(t-T))-u_{n}(-t)(H(t+T)-H(t)), n \in \mathbb{N}$. Hence, $\mathrm{W}_{1}^{n}=\widehat{\Psi} P_{\mathbf{L}_{1}} \mathcal{U}_{n}+\widehat{\Psi} P_{\mathbf{L}_{2}} \mathcal{U}_{n}=\widehat{\Psi} \Psi^{+} \mathrm{W}_{0}^{n}+\widehat{\Psi} P_{\mathbf{L}_{2}} \mathcal{U}_{n}$. Therefore, $\mathrm{W}_{1}^{n}-\Upsilon \mathrm{W}_{0}^{n} \in \widehat{\Psi}(N(\Psi)), n \in \mathbb{N}$. Taking into account Theorem 4.6: (ii), we obtain from here that (4.7) holds.

Sufficiency of (4.7). Denote $\mathrm{W}_{0}^{n}=H\left(n^{2}-x^{2}\right) \mathrm{W}_{0}^{0}, n \in \mathbb{N}$. Obviously, $\left\|\mathrm{W}_{0}^{0}-\mathrm{W}_{0}^{n}\right\|_{0}^{0} \rightarrow 0$ as $n \rightarrow \infty$. Due to the Paley-Wiener theorem, we conclude that $\mathcal{F} W_{0}^{n}$ can be extended to an odd entire function. Hence, $\frac{1}{\sqrt{|\sigma|}} \mathcal{F} \mathrm{W}_{0}^{n} \in H_{1 / 2}^{0}$. According to Theorem 4.3: (i), we have $\mathrm{W}_{0}^{n} \in R(\Psi), n \in \mathbb{N}$. For each $n \in \mathbb{N}$ put $\mathcal{U}_{n}^{1}=\Psi^{+} \mathrm{W}_{0}^{n} \in \mathbf{L}_{1}$ and find $\mathcal{U}_{n}^{2} \in N(\Psi)=\mathbf{L}_{2}$ such that $\left\|\mathrm{W}_{1}^{0}-\Upsilon \mathrm{W}_{0}^{0}-\widehat{\Psi} \mathcal{U}_{n}^{2}\right\|_{0}^{-1} \rightarrow 0$ as $n \rightarrow \infty$. Put $\mathcal{U}_{n}=\mathcal{U}_{n}^{1}+\mathcal{U}_{n}^{2}, \mathrm{~W}_{1}^{n}=\widehat{\Psi} \mathcal{U}_{n}, \mathrm{~W}^{n}=\left(\begin{array}{c}\mathrm{W}_{0}^{n} \\ \mathrm{~W}_{1}^{n}\end{array}\right), n \in \mathbb{N}$. We have $\mathrm{W}_{0}^{n}=\widehat{\Psi} \mathfrak{U}_{n}^{1}=\widehat{\Psi} \mathfrak{U}_{n}, \mathrm{~W}_{1}^{n}=\Upsilon \mathrm{W}_{0}^{n}+\widehat{\Psi} \mathfrak{U}_{n}^{2}, n \in \mathbb{N}$. With regard to Theorem 4.6: (ii), we get

$$
\left\|\mathrm{W}^{0}-\mathrm{W}^{n}\right\| \mid \rightarrow 0 \quad \text { as } n \rightarrow \infty,
$$

where $\mathrm{W}^{n}=\left(\begin{array}{c}\Psi \mathcal{U}_{n} \\ \widehat{\Psi} \mathcal{U}_{n}\end{array}\right), n \in \mathbb{N}$. For each $n \in \mathbb{N}$ consider a sequence $\left\{\mathcal{U}_{n}^{k}\right\}_{k \geq 2} \subset \mathrm{L}^{\infty}(\mathbb{R})$ such that supp $\mathcal{U}_{n}^{k} \subset$ $[-k, k], k \geq 2$, and $\left\|\mathfrak{U}_{n}-\mathfrak{U}_{n}^{k}\right\|_{0}^{0} \rightarrow 0$ as $k \rightarrow \infty$. Determine $k_{n} \geq 2$ such that $\left\|\mathcal{U}_{n}-\mathfrak{U}_{n}^{k_{n}}\right\|_{0}^{0}<\left\|\mathrm{W}^{0}-\mathrm{W}^{n}\right\| \|$, $n \in \mathbb{N}$. Theorems 4.3: (ii), 4.4: (ii) and (4.8) imply

$$
\left\|\left|\mathrm{W}^{0}-\left(\begin{array}{c}
\Psi \mathcal{U}_{n}^{k_{n}} \\
\widehat{\Psi} \mathcal{U}_{n}^{k_{n}}
\end{array}\right)\left\|\leq\left|\left\|\mathrm{W}^{0}-\mathrm{W}^{n}\right\|\right|+\sqrt{2}\right\| \mathcal{U}_{n}-\mathcal{U}_{n}^{k_{n}}\left\|_{0}^{0} \leq(1+\sqrt{2})\right\|\left\|\mathrm{W}^{0}-\mathrm{W}^{n}\right\|\right| \rightarrow 0 \quad \text { as } n \rightarrow \infty .\right.
$$

Using Lemma A.12 and $\left(3.5^{\prime}\right)$, we obtain that for the solution $\mathrm{W}$ to $(3.2),(3.3)$ we have $\left\|\mid \mathrm{W}\left(\cdot, k_{n}\right)\right\| \| \rightarrow 0$ as $n \rightarrow \infty$. The theorem is proved.

According to Theorem 5.10 (see below Sect. 5), we have $\overline{\widehat{\Psi}(N(\Psi))}=\widetilde{H}_{0}^{-1}$ (the closure is considered in $\widetilde{H}_{0}^{-1}$ ). Hence the following corollary is true.

Corollary 4.9. Let $q>0$. Each state $\mathrm{W}^{0} \in \widetilde{\mathbf{H}}$ is approximately $\mathrm{L}^{\infty}$-controllable at a free time, i.e. system (3.2), (3.3) is approximately $\mathrm{L}^{\infty}$-controllable.

In the case $q>0$ the behavior of system (3.2), (3.3) essentially differs from its behavior in the case $q=0$. This difference is generated by the properties of the influence operator $\Psi$. Indeed, $N(\Psi)=\{0\}$ and $\widehat{\Psi}(N(\Psi))=\{0\}$ if $q=0$, and $N(\Psi) \backslash\{0\} \neq \emptyset$ and $\widehat{\Psi}(N(\Psi))=\widetilde{H}_{0}^{-1}$ otherwise.

Remark 4.10. Let $\mathrm{W}^{0} \in \widetilde{\mathbf{H}}$ and supp $\mathrm{W}^{0} \subset[-\alpha, \alpha], \alpha>0$. With regard to Corollaries 3.9, 4.9 and Theorems 4.7, 4.8, we obtain the following assertions.

(i) In the case $q=0$ a state $\mathrm{W}^{0}$ is approximately $\mathrm{L}^{\infty}$-controllable at a free time iff condition (3.8) (or (4.6)) holds, and under this condition the state $\mathrm{W}^{0}$ is $\mathrm{L}^{\infty}$-controllable at a given time $T \geq \alpha$. 
(ii) In the case $q>0$ a state $\mathrm{W}^{0}$ is always approximately $\mathrm{L}^{\infty}$-controllable at a free time, but it is (approximately) $\mathrm{L}^{\infty}$-controllable at a given time $T \geq \alpha$ iff condition (3.8) holds.

The case (ii) is illustrated by Example 7.6.

By analogy with the operators $\Psi^{+}$and $\Upsilon$, we introduce the "symmetric" operators $\widehat{\Psi}^{+}$and $\widehat{\Upsilon}$. Decompose again the space $\widetilde{H}_{0}^{0}$ into a direct sum of subspaces. Denote $\mathbf{M}_{1}=(N(\widehat{\Psi}))^{\perp}, \mathbf{M}_{2}=N(\widehat{\Psi})$. Evidently, $\mathbf{M}_{1}=$ $\left\{g \in \widetilde{H}_{0}^{0} \mid \operatorname{supp} \mathcal{F}(\operatorname{sgn} t g) \subset \mathbb{R} \backslash(-q, q)\right\}, \mathbf{M}_{2}=\left\{g \in \widetilde{H}_{0}^{0} \mid \operatorname{supp} \mathcal{F}(\operatorname{sgn} t g) \subset[-q, q]\right\}$. We have $\widetilde{H}_{0}^{0}=\mathbf{M}_{1} \bigoplus \mathbf{M}_{2}$. Taking into account Theorem 4.4, we conclude that the restriction $\left.\widehat{\Psi}\right|_{\mathbf{M}_{1}}$ is an invertible operator. Denote $\widehat{\Psi}^{+}=\left(\left.\widehat{\Psi}\right|_{\mathbf{M}_{1}}\right)^{-1}, D\left(\widehat{\Psi}^{+}\right)=R(\widehat{\Psi})$. Here $\widehat{\Psi}^{+}$is the Moore-Penrose inverse for $\widehat{\Psi}$. We have $\widehat{\Psi} \widehat{\Psi}^{+}=\operatorname{Id}$, $\widehat{\Psi}^{+} \widehat{\Psi}=P_{\mathbf{M}_{1}}$, where $P_{\mathbf{M}_{j}}$ is the projector on $\mathbf{M}_{j}$ in $\widetilde{H}_{0}^{0}, j=1,2$. One can see that

$$
\widehat{\Psi}^{+} f=\operatorname{sgn} t \mathcal{F}_{\mu \rightarrow t}^{-1}\left(\frac{-i H\left(\mu^{2}-q^{2}\right)}{\sqrt{\mu^{2}-q^{2}}}(\mathcal{F} f)\left(\sqrt{\mu^{2}-q^{2}}\right)\right), \quad f \in D\left(\widehat{\Psi}^{+}\right) .
$$

Denote $\widehat{\Upsilon}: H_{0}^{-1} \rightarrow H_{0}^{0}, D(\widehat{\Upsilon})=\widetilde{H}_{0}^{-1}$,

$$
\widehat{\Upsilon} f=\operatorname{sgn} x \mathcal{F}_{\sigma \rightarrow x}^{-1}\left(\frac{(\mathcal{F} f)(\sigma)}{i \operatorname{sgn} \sigma \sqrt{\sigma^{2}+q^{2}}}\right), \quad f \in D(\widehat{\Upsilon}) .
$$

With regard to Lemma A.17, we have $\widehat{\Upsilon} f=\Psi \widehat{\Psi}^{+} f, f \in R(\widehat{\Psi})$.

By analogy with Theorem 4.5, we obtain

Theorem 4.11. Let $q>0$. Then the following assertions hold:

(i) $R\left(\widehat{\Psi}^{+}\right)=\mathbf{M}_{1}$;

(ii) $\widehat{\Psi}^{+}$is invertible, $\left(\widehat{\Psi}^{+}\right)^{-1}=\left.\widehat{\Psi}\right|_{\mathbf{M}_{1}}, D\left(\left(\widehat{\Psi}^{+}\right)\right)^{-1}=R\left(\widehat{\Psi}^{+}\right)=\mathbf{M}_{1}$;

(iii) $\left(\widehat{\Psi}^{+} f\right)(t)=\operatorname{sgn} t \tilde{f}(t)-q \int_{0}^{|t|} \frac{J_{1}\left(q \sqrt{t^{2}-x^{2}}\right)}{\sqrt{t^{2}-x^{2}}} \tilde{f}(x) \mathrm{d} x, \tilde{f}=\mathcal{F}^{-1}\left(\frac{(\mathcal{F} f)(\sigma)}{\operatorname{isgn} \sigma \sqrt{\sigma^{2}+q^{2}}}\right)=\operatorname{sgn} x \widehat{\Upsilon} f=$ $\frac{1}{2} f(x) *\left(\operatorname{sgn} x I_{0}(q x)-L_{0}(q x)\right), f \in R(\widehat{\Psi}) ;$

To obtain (iii), we use (A.8) and [4], (3.11). Taking into account the definition of $\Upsilon$, Lemmas A.17, A.18, and Corollary A.19, we obtain

Theorem 4.12. Let $q>0$. Then the following assertions hold:

(i) $R(\widehat{\Upsilon})=\widetilde{H}_{0}^{0}$;

(ii) $\widehat{\Upsilon}$ is bounded and $\|\widehat{\Upsilon}\| \leq \frac{\sqrt{1+q^{2}}}{q}$;

(iii) $\widehat{\Upsilon}$ is invertible, $\widehat{\Upsilon}^{-1} g=\mathcal{F}_{\sigma \rightarrow x}^{-1}\left(\mathrm{i} \operatorname{sgn} \sigma \sqrt{\sigma^{2}+q^{2}}(\mathcal{F}(\operatorname{sgn} \xi g))(\sigma)\right), g \in D\left(\widehat{\Upsilon}^{-1}\right)=R(\widehat{\Upsilon})=\widetilde{H}_{0}^{0}$;

(iv) $\widehat{\Upsilon}^{-1}$ is bounded, $\left\|\widehat{\Upsilon}^{-1}\right\| \leq \sqrt{1+q^{2}}$;

(v) $\widehat{\Upsilon} f=\frac{1}{2} \operatorname{sgn} x\left(f(x) *\left(\operatorname{sgn} x I_{0}(q x)-L_{0}(q x)\right)\right), f \in \widetilde{H}_{0}^{-1}$.

By analogy with Theorem 4.8, we obtain

Theorem 4.13. A state $\mathrm{W}^{0} \in \widetilde{\mathbf{H}}$ is approximately $\mathrm{L}^{\infty}$-controllable at a free time iff

$$
\mathrm{W}_{0}^{0}-\widehat{\Upsilon} \mathrm{W}_{1}^{0} \in \overline{\Psi(N(\widehat{\Psi}))}
$$

where the closure is considered with respect to the norm $\|\cdot\|_{0}^{0}$. 
Remark 4.14. Theorems 4.8 and 4.13 give us a practical method for solving the approximate $\mathrm{L}^{\infty}$-controllability problem at a free time for system (3.2), (3.3). Let us split the initial state $\mathrm{W}^{0} \in \widetilde{\mathbf{H}}$ :

$$
\mathrm{W}^{0}=\left(\begin{array}{c}
\mathrm{W}_{0}^{0} \\
\mathrm{~W}_{1}^{0}
\end{array}\right)=\left(\begin{array}{c}
\mathrm{W}_{0}^{0} \\
0
\end{array}\right)+\left(\begin{array}{c}
0 \\
\mathrm{~W}_{1}^{0}
\end{array}\right) .
$$

With regard to Theorems 5.10, 5.12, we can find sequences $\left\{\mathcal{U}_{n}^{s}\right\}_{n=0}^{\infty} \subset \mathrm{L}^{\infty}(\mathbb{R}), \mathcal{U}_{n}^{s}$ is odd, $n=\overline{0, \infty}, s=0,1$, such that

$$
\begin{aligned}
& \mathcal{U}_{n}^{0} \in N(\widehat{\Psi}), \quad n=\overline{0, \infty}, \quad \text { and } \quad \Psi \mathcal{U}_{n}^{0} \rightarrow \mathrm{W}_{0}^{0}=\mathrm{W}_{0}^{0}-\widehat{\Upsilon} 0 \quad \text { as } n \rightarrow \infty \text {; } \\
& \mathcal{U}_{n}^{1} \in N(\Psi), \quad n=\overline{0, \infty}, \quad \text { and } \quad \widehat{\Psi} \mathcal{U}_{n}^{1} \rightarrow \mathrm{W}_{1}^{0}=\mathrm{W}_{1}^{0}-\Upsilon 0 \quad \text { as } n \rightarrow \infty .
\end{aligned}
$$

Therefore, for $\mathcal{U}_{n}=\mathcal{U}_{n}^{0}+\mathcal{U}_{n}^{1}, n=\overline{0, \infty}$, we have

$$
\Psi \mathcal{U}_{n} \rightarrow \mathrm{W}_{0}^{0} \quad \widehat{\Psi} \mathcal{U}_{n} \rightarrow \mathrm{W}_{1}^{0} \quad \text { as } n \rightarrow \infty .
$$

Choosing an appropriate approximation of $\mathcal{U}_{n}$ by a function of the form $\mathfrak{U}_{n}^{k}=(H(t+k)-H(t-k)) \mathcal{U}_{n}, k=\overline{2, \infty}$, $n=\overline{0, \infty}$, we get a solution of the approximate $\mathrm{L}^{\infty}$-controllability problem at a free time for system (3.2), (3.3).

\section{Properties of the Sets $N(\Psi), \widehat{\Psi}(N(\Psi))$ And $N(\widehat{\Psi}), \Psi(N(\widehat{\Psi}))$}

According to Theorems 4.8 and 4.13, investigation of conditions (4.7) and (4.10) is a key point in the study of the approximate $L^{\infty}$-controllability problem for the wave equation on a half-axis at a free time. That is why we investigate the sets $N(\Psi), \widehat{\Psi}(N(\Psi))$ and $N(\widehat{\Psi}), \Psi(N(\widehat{\Psi}))$ here. Since for $q=0$ we have $N(\Psi)=\widehat{\Psi}(N(\Psi))=N(\widehat{\Psi})=\Psi(N(\widehat{\Psi}))=\{0\}$, we suppose $q>0$ throughout the section. Theorems 5.10 and 5.12 are the main results of this section. They assert that $\widetilde{H}_{0}^{-1}$ is the closure of $\widehat{\Psi}(N(\Psi))$ in $H_{0}^{-1}$, and $\widetilde{H}_{0}^{0}$ is the closure of $\Psi(N(\widehat{\Psi}))$ in $H_{0}^{0}$.

Applying the Paley-Wiener theorem, Theorems 4.3: (iii), 4.4: (iii), the definitions of $\Psi$ and $\widehat{\Psi}$, we obtain two theorems.

Theorem 5.1. The following four assertions are equivalent

(i) $g \in N(\Psi)$;

(ii) $\forall n=\overline{0, \infty} g^{(n)} \in N(\Psi)$.

(iii) $g \in \widetilde{H}_{0}^{0}$ and $\operatorname{supp} \mathcal{F} g \subset[-q, q]$;

(iv) $g \in \widetilde{H}_{0}^{0}$ and $g$ can be extended to an entire function of the order $\leq 1$ and the type $\leq q$.

Theorem 5.2. The following four assertions are equivalent

(i) $g \in N(\widehat{\Psi})$;

(ii) $\forall n=0,1 g^{(n)} \in N(\widehat{\Psi})$.

(iii) $g \in \widetilde{H}_{0}^{0}$ and $\operatorname{supp} \mathcal{F}(\operatorname{sgn} t g) \subset[-q, q]$;

(iv) $g \in \widetilde{H}_{0}^{0}$ and $\operatorname{sgn} t g$ can be extended to an entire function of the order $\leq 1$ and the type $\leq q$.

The following two theorems give us properties of $\widehat{\Psi}(N(\Psi))$.

Theorem 5.3. The following assertions hold

(i) $R\left(\left.\widehat{\Psi}\right|_{N(\Psi)}\right) \subset \widetilde{H}_{0}^{0} \subset \widetilde{H}_{0}^{-1}$;

(ii) $N\left(\left.\widehat{\Psi}\right|_{N(\Psi)}\right)=\{0\}$. 
Proof. (i) If $g \in N(\Psi)$, then $(\operatorname{sgn} t g)^{\prime}=\operatorname{sgn} t g^{\prime} \in \widetilde{H}_{0}^{0}$ because of Theorem 5.1: (i), (ii). According to Theorem 4.3: (i), we get $\widehat{\Psi} g=\Psi\left((\operatorname{sgn} t g)^{\prime}\right) \in \widetilde{H}_{0}^{0}$.

(ii) Let $G=\mathcal{F}(\operatorname{sgn} t g), g \in N(\Psi)$ and $\widehat{\Psi} g=0$. Then $G(\sigma)=0,|\sigma|>q$. Taking into account Theorem 5.1: (i), (iii), we conclude that $G(\sigma)=\int_{-q}^{q}(\mathcal{F} g)(\xi) \frac{\mathrm{d} \xi}{\sigma-\xi}$ is holomorphic on $\mathbb{C} \backslash[-q, q]$. Therefore, $G=0$. Hence, $g=0$. The theorem is proved.

Theorem 5.4. Let $f \in \widetilde{H}_{0}^{-1}$. Then $f \in \widehat{\Psi}(N(\Psi))$ iff there exists $\Phi \in H_{0}^{0}$ such that

(i) $\operatorname{supp} \Phi \subset[0, q]$;

(ii) $\xi^{-1 / 2}\left(q^{2}-\xi^{2}\right)^{-1 / 4} \Phi \in H_{0}^{0}$;

(iii) $f=\frac{2}{\pi} \mathcal{F}_{\sigma \rightarrow x}^{-1}\left(\sigma \int_{0}^{q} \frac{\Phi(\xi) \mathrm{d} \xi}{\sigma^{2}+\xi^{2}}\right)=\mathrm{i} \operatorname{sgn} x \sqrt{\frac{2}{\pi}} \int_{0}^{q} \Phi(\xi) \mathrm{e}^{-\xi|x|} \mathrm{d} \xi$.

Moreover, under conditions (i)-(iii) we have $\widehat{\Psi} g=f$ for $g=\mathcal{F}_{\xi \rightarrow t}^{-1}\left(\operatorname{sgn} \xi \Phi\left(\sqrt{q^{2}-\xi^{2}}\right) / \sqrt{q^{2}-\xi^{2}}\right) \in N(\Psi)$.

Proof. Sufficiency of (i)-(iii). Put $G(\xi)=\operatorname{sgn} \xi \Phi\left(\sqrt{q^{2}-\xi^{2}}\right) / \sqrt{q^{2}-\xi^{2}}$. According to (i) and (ii), we have $\operatorname{supp} G \subset[-q, q]$ and $G \in \widetilde{H}_{0}^{0}$. Due to Theorem 5.1: (i), (iii), we get $g=\mathcal{F} G \in N(\Psi)$. With regard to (4.2), we obtain

$$
\begin{aligned}
\widehat{\Psi} g & =\frac{1}{\pi} \mathcal{F}_{\sigma \rightarrow x}^{-1}\left(\sigma\left(G(\nu) * \frac{1}{\nu}\right)\left(\sqrt{\sigma^{2}+q^{2}}\right)\right) \\
& =\frac{2}{\pi} \mathcal{F}_{\sigma \rightarrow x}^{-1}\left(\sigma \int_{0}^{q} \frac{\mu G(\mu) \mathrm{d} \mu}{\sigma^{2}+q^{2}-\mu^{2}}\right)=\frac{2}{\pi} \mathcal{F}_{\sigma \rightarrow x}^{-1}\left(\sigma \int_{0}^{q} \frac{\xi G\left(\sqrt{q^{2}-\xi^{2}}\right)}{\sigma^{2}+\xi^{2}} \mathrm{~d} \xi\right)
\end{aligned}
$$

Then (iii) implies $f \in \widehat{\Psi}(N(\Psi))$.

Necessity of (i)-(iii). If $f \in \widehat{\Psi}(N(\Psi))$, then there exists $g \in N(\Psi)$ such that $f=\widehat{\Psi} g$. Setting $G=\mathcal{F} g$ and applying Theorem 5.1: (i), (iii), we conclude that $\operatorname{supp} G \subset[-q, q], G \in \widetilde{H}_{0}^{0}$. With regard to (5.1), we get $f=\widehat{\Psi} g=\frac{2}{\pi} \mathcal{F}_{\sigma \rightarrow x}^{-1}\left(\sigma \int_{0}^{q} \frac{\mu G(\mu) \mathrm{d} \mu}{\sigma^{2}+q^{2}-\mu^{2}}\right)$. Put $\Phi(\xi)=H(\xi) \xi G\left(\sqrt{q^{2}-\xi^{2}}\right)$. Then (i)-(iii) are true. The theorem is proved.

Analogously, we obtain properties of $\Psi(N(\widehat{\Psi}))$.

Theorem 5.5. The following assertions hold

(i) $R\left(\left.\Psi\right|_{N(\widehat{\Psi})}\right) \subset \widetilde{H}_{0}^{0}$;

(ii) $N\left(\left.\Psi\right|_{N(\widehat{\Psi})}\right)=\{0\}$.

Theorem 5.6. Let $f \in \widetilde{H}_{0}^{0}$. Then $f \in \Psi(N(\widehat{\Psi}))$ iff there exists $\Phi \in H_{0}^{0}$ such that

(i) $\operatorname{supp} \Phi \subset[0, q]$;

(ii) $\xi^{-1 / 2}\left(q^{2}-\xi^{2}\right)^{1 / 4} \Phi \in H_{0}^{0}$;

(iii) $f=\frac{2}{\pi} \mathcal{F}_{\sigma \rightarrow x}^{-1}\left(\sigma \int_{0}^{q} \frac{\Phi(\xi) \mathrm{d} \xi}{\sigma^{2}+\xi^{2}}\right)=\mathrm{i} \operatorname{sgn} x \sqrt{\frac{2}{\pi}} \int_{0}^{q} \Phi(\xi) \mathrm{e}^{-\xi|x|} \mathrm{d} \xi$.

Moreover, under conditions (i)-(iii) we have $\Psi g=f$ for $g=\operatorname{sgn} t \mathcal{F}_{\xi \rightarrow t}^{-1}\left(\mathrm{i}|\xi| \Phi\left(\sqrt{q^{2}-\xi^{2}}\right) / \sqrt{q^{2}-\xi^{2}}\right) \in N(\widehat{\Psi})$.

Remark 5.7. Condition (iii) in Theorems 5.4 and 5.6 can be represented in the form $(\mathcal{F} f)(\sigma)=\frac{1}{\pi}\langle\Phi, \nu(\sigma, \cdot)\rangle$ where $\nu(\sigma, \xi)=\frac{2 \sigma}{\sigma^{2}+\xi^{2}}, \sigma \in \mathbb{R}, \xi \in \mathbb{R}$.

With regard to Theorems 5.4 and 5.6, we see that the following theorem is useful for investigation of $(\Psi N(\widehat{\Psi}))$ and $\widehat{\Psi}(N(\Psi))$. Due to [7], Chapter 2, the Paley-Wiener Theorem, and Lemma A.20, we get 
Theorem 5.8. Let $\Phi \in H_{0}^{0}$ and $\operatorname{supp} \Phi \in[0, q]$. Then $f(x)=\mathrm{i} \operatorname{sgn} x \sqrt{\frac{2}{\pi}} \int_{0}^{q} \Phi(\xi) \mathrm{e}^{-\xi|x|} \mathrm{d} \xi, x>0$ iff

(i) $f \in L^{2}(0,+\infty)$;

(ii) $f$ can be extended to an entire function and $\mathrm{e}^{-z q / 2} f(z)$ is of the order $\leq 1$ and the type $\leq q / 2$;

(iii) $\forall x>0 f_{\circledast} \in H_{0}^{0}$, where $f_{\circledast}(y)=f(x+i y), y \in \mathbb{R}$;

(iv) $\sup \left\{\left\|f_{\circledast}\right\|_{0}^{0} \mid x>0\right\} \leq\left\|f_{\odot}\right\|_{0}^{0}$.

Under conditions (i)-(iv) we have $f_{(\mathbb{D}}=2 i \mathcal{F}_{\xi \rightarrow y} \Phi$.

The following two theorems give us properties of $\overline{\Psi(N(\Psi))}$.

Theorem 5.9. Let $n=\overline{0, \infty}$. Then $\operatorname{sgn} x|x|^{n} \mathrm{e}^{-q|x|} \in \overline{\widehat{\Psi}(N(\Psi))}$ (the closure is considered in $H_{0}^{-1}$ ). Proof. Let $n=\overline{0, \infty}$ be fixed. Put $\nu(\sigma, \xi)=\frac{2 \sigma}{\sigma^{2}+\xi^{2}}, \sigma, \xi \in \mathbb{R}, f(x)=\operatorname{sgn} x|x|^{n} \mathrm{e}^{-q|x|}, x \in \mathbb{R}$. We have

$$
F(\sigma)=\left(\mathcal{F}_{x \rightarrow \sigma} f\right)(\sigma)=-\mathrm{i} \sqrt{\frac{1}{2 \pi}}\left\langle(-1)^{n} \delta^{(n)}(\xi-q), \nu(\sigma, \xi)\right\rangle, \quad \sigma \in \mathbb{R}
$$

Let $\mu \in C^{\infty}(\mathbb{R}), \operatorname{supp} \mu \subset[-1,1], \int_{-1}^{1} \mu(\xi) \mathrm{d} \xi=1$ and $\mu \geq 0, \xi \in \mathbb{R}$. Put $\mu_{m}=m \mu(m(\xi-q)+2)$, $\xi \in \mathbb{R}, m \in \mathbb{N}$. Then $\mu_{m} \in C^{\infty}(\mathbb{R})$ and $\operatorname{supp} \mu_{m} \subset[q-3 / m, q-1 / m], m \in \mathbb{N}$. For $m \in \mathbb{N}$ set $F_{m}(\sigma)=$ $-\mathrm{i} \sqrt{\frac{1}{2 \pi}}\left\langle(-1)^{n} \mu_{m}^{(n)}, \nu(\sigma, \cdot)\right\rangle, \sigma \in \mathbb{R}$. With regard to Theorem 5.4, we conclude that $f_{m}=\mathcal{F}_{\sigma \rightarrow x}^{-1} F_{m} \in \widehat{\Psi}(N(\Psi))$, $m \in \mathbb{N}$. Set $\widehat{\mu}(\xi)=\int_{-\infty}^{\xi} \mu(u) \mathrm{d} u, \xi \in \mathbb{R}$. Then $0 \leq \widehat{\mu}(\xi) \leq 1$ for $\xi \in \mathbb{R}, \widehat{\mu}(\xi)=0$ for $\xi \leq-1$ and $\widehat{\mu}(\xi)=1$ for $\xi \geq 1$. Put $\widehat{\mu}_{m}=\widehat{\mu}(m(\xi-q)+2), \xi \in \mathbb{R}, m \in \mathbb{N}$. Evidently, $\left(\widehat{\mu}_{m}\right)^{\prime}=\mu_{m}, m \in \mathbb{N}$. We have $\left\|H(\xi-q)-\widehat{\mu}_{m}\right\|_{0}^{0} \leq\left(\int_{q-3 / m}^{q-1 / m} \widehat{\mu}_{m}(\xi) \mathrm{d} \xi\right)^{1 / 2} \leq \sqrt{\frac{2}{m}}, m \in \mathbb{N}$. Therefore, $\left\|\delta^{(n)}(\xi-q)-\mu_{m}^{(n)}\right\|_{0}^{-2 n-2} \leq$ $\sqrt{\frac{2}{m}}$. Hence, $\left\|\xi^{-2 n-2}\left(\mu_{m}^{(n)}-\delta^{(n)}(\xi-q)\right)\right\|_{2 n+2}^{-2 n-2} \leq \sqrt{\frac{2}{m}}\left(1+\frac{1}{(q-3 / m)^{2}}\right)^{n+1}, m \in \mathbb{N}$. Taking into account Lemma A.20 and (5.2), we conclude that

$$
\left\|F-F_{m}\right\|_{0}^{0} \leq L_{2 n+2} \sqrt{\frac{\pi}{m}}\left(1+\frac{1}{(q-3 / m)^{2}}\right)^{n+1} \rightarrow 0 \quad \text { as } m \rightarrow \infty .
$$

Therefore, $f_{m} \rightarrow f$ as $m \rightarrow \infty$ in $H_{0}^{-1}$. Since $f_{m}=\mathcal{F}_{\sigma \rightarrow x}^{-1} F_{m} \in \widehat{\Psi}(N(\Psi)), m \in \mathbb{N}$, then $f$ belongs to the closure $\widehat{\Psi}(N(\Psi))$ in $H_{0}^{-1}$. That was to be proved.

Theorem 5.10. $\widetilde{H}_{0}^{-1}$ is the closure of $\widehat{\Psi}(N(\Psi))$ with respect to to the norm $\|\cdot\|_{0}^{-1}$.

Proof. Let $f \in \widetilde{H}_{0}^{-1}$. Due to [7], Chapter $1, \widetilde{H}_{0}^{-1}$ is the closure of $\widetilde{H}_{0}^{0}$ with respect to the norm $\|\cdot\|_{0}^{-1}$. Since $\left\{\operatorname{sgn} x|x|^{n} \mathrm{e}^{-q|x|}\right\}_{n=0}^{\infty}$ is a basis of $\widetilde{H}_{0}^{0}$, then $f$ can be approximated in $\widetilde{H}_{0}^{-1}$ by the functions of the form $f_{N}(x)=\operatorname{sgn} x \mathrm{e}^{-q|x|} \sum_{n=0}^{N} f_{n}^{N}|x|^{n}$, where $f_{n}^{N} \in \mathbb{R}, n=\overline{0, N}, N=\overline{0, \infty}$. Taking into account Theorem 5.9, we conclude that $f$ belongs to the closure of $\widehat{\Psi}(N(\Psi))$ with respect to the norm $\|\cdot\|_{0}^{-1}$. That was to be proved.

Analogously, we obtain properties of $\overline{\Psi(N(\widehat{\Psi}))}$.

Theorem 5.11. Let $n=\overline{0, \infty}$. Then $\operatorname{sgn} x|x|^{n} \mathrm{e}^{-q|x|} \in \overline{\Psi(N(\widehat{\Psi}))}$ (the closure is considered in $\left.H_{0}^{0}\right)$.

Theorem 5.12. $\widetilde{H}_{0}^{0}$ is the closure of $\Psi(N(\widehat{\Psi}))$ with respect to to the norm $\|\cdot\|_{0}^{0}$.

Remark 5.13. Let $\mathcal{S}_{0}=\left\{\varphi \in \mathcal{S} \mid \varphi^{(k)}(0)=0, k=\overline{0, \infty}\right\}, \mathcal{S}^{0}=\mathcal{F S}_{0}$. By formulae (4.1) and (4.2), the operators $\Psi$ and $\widehat{\Psi}$ can be extended on $\mathcal{S}^{0^{\prime}}$. Denote these extensions as $\Psi_{\infty}$ and $\widehat{\Psi}_{\infty}$ respectively. We have

$$
\left(\mathcal{F}_{x \rightarrow \sigma}\left(\operatorname{sgn} x|x|^{n} \mathrm{e}^{-q|x|}\right)\right)(\sigma)=\sqrt{\frac{2}{\pi}} \sum_{p=0}^{n} A_{p}^{n} \frac{\sigma}{\left(\sigma^{2}+q^{2}\right)^{p+1}}, \quad \sigma \in \mathbb{R},
$$


where $A_{p}^{n} \in \mathbb{C}, p=\overline{0, n}, n=\overline{0, \infty}$. Therefore,

$$
\operatorname{sgn} x|x|^{n} \mathrm{e}^{-q|x|}=\Psi_{\infty}\left(i \sum_{p=0}^{n} A_{p}^{n} \frac{(-1)^{p}}{(2 p) !} t^{2 p} \operatorname{sgn} t\right)=\widehat{\Psi}_{\infty}\left(\sum_{p=0}^{n} A_{p}^{n} \frac{(-1)^{p+1}}{(2 p+1) !} t^{2 p+1}\right) .
$$

\section{Conditions for approximate $\mathcal{B}^{U}$-COntrollability at a free time}

Consider control system (1.1), (1.2), (3.1) and equivalent system (3.2), (3.3) where $T>0$ is a parameter.

Definition 6.1. Let $U>0$. A state $\mathrm{W}^{0} \in \widetilde{\mathbf{H}}$ is called approximately $\mathcal{B}^{U}$-controllable at a free time if 0 belongs to the closure of $\bigcup_{T>0} \mathcal{R}_{T}^{U}\left(\mathrm{~W}^{0}\right)$ in $\widetilde{\mathbf{H}}$.

In [15] Theorem 1.1, the following assertion was proved.

Theorem 6.2. Let $q=0$. A state $\mathrm{W}^{0} \in \widetilde{\mathbf{H}}$ is approximately $\mathcal{B}^{U}$-controllable at a free time iff $\left\|\mathrm{W}_{0}^{0}\right\|_{\mathrm{L}^{\infty}(\mathbb{R})} \leq U$ and condition (4.6) holds.

Let $q>0$. Analyzing the proof of Theorem 4.8 and Examples 7.3-7.9, we see that the approximate $\mathcal{B}^{U_{-}}$ controllability problem at a free time is essentially more complicated than the approximate $\mathrm{L}^{\infty}$-controllability problem at a free time. Moreover, the method that allows us to solve the second of these problems is not applicable to the first one. However, using the results of Section 5, we can obtain a sufficient condition for approximate $\mathcal{B}^{U}$-controllability at a free time.

Theorem 6.3. Let $q>0, \mathrm{~W}_{0}^{0} \in \Psi(N(\widehat{\Psi})), \mathrm{W}_{1}^{0} \in \widehat{\Psi}(N(\Psi))$. Then for $\mathrm{W}_{0}^{0}$ and $\mathrm{W}_{1}^{0}$ conditions (i)-(iv) of Theorem 5.8 are valid and the state $\mathrm{W}^{0}=\left(\begin{array}{c}\mathrm{W}_{0}^{0} \\ \mathrm{~W}_{1}^{0}\end{array}\right)$ is approximately $\mathcal{B}^{U}$-controllable at a free time for

$$
U \geq \frac{1}{\sqrt{2 \pi}}\left(\left\|\mathcal{F}\left(\mathrm{W}_{0}^{0}\right)_{\mathbb{Q}}\right\|_{L^{1}(\mathbb{R})}+\left\|\mathcal{F}\left(\left(\mathrm{W}_{1}^{0}\right)_{\mathbb{Q}}\right) / \sqrt{q^{2}-\xi^{2}}\right\|_{L^{1}(\mathbb{R})}\right)
$$

Proof. Taking into account Theorems 5.4, 5.6, 5.8, put

$$
\begin{array}{lll}
\Phi_{0}=-\frac{\mathrm{i}}{2} \mathcal{F}\left(\mathrm{W}_{0}^{0}\right)_{\mathbb{(})}, & g_{0}=\operatorname{sgn} t \mathcal{F}_{\xi \rightarrow t}^{-1}\left(\mathrm{i}|\xi| \frac{\Phi_{0}\left(\sqrt{q^{2}-\xi^{2}}\right)}{\sqrt{q^{2}-\xi^{2}}}\right) \in N(\widehat{\Psi}), & \Psi g_{0}=\mathrm{W}_{0}^{0} ; \\
\Phi_{1}=-\frac{\mathrm{i}}{2}\left(\mathcal{F}\left(\mathrm{W}_{1}^{0}\right)_{(}\right), & g_{1}=\mathcal{F}_{\xi \rightarrow t}^{-1}\left(\operatorname{sgn} \xi \frac{\Phi_{1}\left(\sqrt{q^{2}-\xi^{2}}\right)}{\sqrt{q^{2}-\xi^{2}}}\right) \in N(\Psi), & \widehat{\Psi} g_{1}=\mathrm{W}_{1}^{0} .
\end{array}
$$

Applying again these theorems, we conclude that $\left\|g_{0}\right\|_{L^{\infty}(\mathbb{R})} \leq \sqrt{\frac{2}{\pi}}\left\|\Phi_{0}\right\|_{L^{1}(\mathbb{R})}$ and $\left\|g_{1}\right\|_{L^{\infty}(\mathbb{R})} \leq \sqrt{\frac{2}{\pi}}$ $\left\|\Phi_{1} / \sqrt{q^{2}-\xi^{2}}\right\|_{L^{1}(\mathbb{R})}$. Therefore, for $\mathcal{U}=g_{0}+g_{1}$ we have $\mathcal{U} \in \widetilde{H}_{0}^{0}, \mathcal{U}$ is odd, $\Psi \mathcal{U}=\mathrm{W}_{0}^{0}, \widehat{\Psi} \mathcal{U}=\mathrm{W}_{1}^{0}$, and $\|\mathcal{U}\|_{L^{\infty}(\mathbb{R})} \leq \sqrt{\frac{2}{\pi}}\left(\left\|\Phi_{0}\right\|_{L^{1}(\mathbb{R})}+\left\|\Phi_{1} / \sqrt{q^{2}-\xi^{2}}\right\|_{L^{1}(\mathbb{R})}\right)$. Taking into account Theorems 4.3, 4.4 and setting $u_{k}(t)=U(t)(H(t)-H(t-k)), t \in \mathbb{R}, k \in \mathbb{N}$, we obtain controls solving the approximate $\mathcal{B}^{U}$-controllability problem at a free time for the state $\mathrm{W}^{0}$, if $U$ satisfies (6.1). The theorem is proved.

Example 7.3 demonstrates that the conditions of the Theorem 6.3 is not necessary for approximate $\mathcal{B}^{U}$ controllability at a free time. Examples 7.10, 7.10 illustrate Theorem 6.3. In particular, we see that estimate (6.1) is not sharp for each $\mathrm{W}^{0} \in \widetilde{\mathbf{H}}$ satisfying the conditions of Theorem 6.3 (see Ex. 7.11). But there exists $\mathrm{W}^{0} \in \widetilde{\mathbf{H}}$ satisfying the conditions of this theorem such that estimate (6.1) is sharp for $\mathrm{W}^{0}$ (see Ex. 7.10). 


\section{EXAMPLES}

In this section we consider examples illustrating the results of Sections 3-6.

Example 7.1. Let $q>0, T>0, \alpha>0$. Put $f(t)=\alpha t(H(t+T)-H(t-T)), h(x)=\left(\Psi_{T} f\right)(x)=$ $\alpha x J_{0}\left(q \sqrt{T^{2}-x^{2}}\right)(H(x+T)-H(x-T))$. Therefore, $f(t)=\left(\Psi_{T}^{-1} h\right)(t)$. Evidently, $\sup \{|f(x)| \mid x \in(-T, T)\}=$ $\alpha T, \sup \{|h(t)| \mid t \in(-T, T)\}=\alpha T$. Setting $\alpha=U / T$, we conclude that (3.13) is not necessary for (3.9) for each $T>0$. Setting $\alpha=(1+q T) U / T$, we conclude that (3.14) is not sufficient for (3.9) for each $T>0$.

Example 7.2. Let $q>0, \mathrm{~W}_{0}^{0}=x J_{0}\left(\sqrt{1-x^{2}}\right) H\left(1-x^{2}\right), \mathrm{W}_{1}^{0}=\frac{\mathrm{d}}{\mathrm{d} x}\left(H\left(1-x^{2}\right)\left(|x|-q \int_{|x|}^{1} t^{2} \frac{J_{1}\left(q \sqrt{t^{2}-x^{2}}\right)}{\sqrt{t^{2}-x^{2}}} \mathrm{~d} t\right)\right)$. Theorem 3.1: (v) yields $\mathrm{W}_{0}^{0}=\Psi_{1} \mathcal{U}$, where $\mathcal{U}(t)=t H\left(1-t^{2}\right), t \in \mathbb{R}$. With regard to Theorem 3.1: (iii),(v), we obtain

$$
\Psi_{1} \frac{\mathrm{d}}{\mathrm{d} t}\left(\operatorname{sgn} t \Psi_{1}^{-1} \mathrm{~W}_{0}^{0}\right)=\Psi_{1}(\operatorname{sgn} t \mathcal{U})^{\prime}=\frac{\mathrm{d}}{\mathrm{d} x}\left(\mathcal{U}(x) \operatorname{sgn} x-q \int_{|x|}^{\infty} t \frac{J_{1}\left(q \sqrt{t^{2}-x^{2}}\right)}{\sqrt{t^{2}-x^{2}}} \mathrm{~d} t\right)=\mathrm{W}_{1}^{0} .
$$

Due to Theorem 3.5, the state $\mathrm{W}^{0}=\left(\begin{array}{l}\mathrm{W}_{0}^{0} \\ \mathrm{~W}_{1}^{0}\end{array}\right)$ is $\mathcal{B}^{U}$-controllable at a given time $T \geq 1$ for $U \geq 1$. The control $u(t)=\mathcal{U}(t) H(t), t \in \mathbb{R}$, solves the $\mathcal{B}^{U}$-controllability problem at the time $T$ for this state.

With regard to Remark 4.14, we may consider the states of the form $\left(\begin{array}{c}\mathrm{W}_{0}^{0} \\ 0\end{array}\right)$ and $\left(\begin{array}{c}0 \\ \mathrm{~W}_{1}^{0}\end{array}\right)$, without loss of generality, when we investigate approximate $\mathrm{L}^{\infty}$-controllability at a free time.

Example 7.3. Let $q>0, n=\overline{0, \infty}, \mathrm{W}_{0}^{0}=|x|^{n} \mathrm{e}^{-q|x|} \operatorname{sgn} x, \mathrm{~W}_{1}^{0}=0$. Due to Corollary 4.9 , the state $\mathrm{W}^{0}=$ $\left(\begin{array}{l}\mathrm{W}_{0}^{0} \\ \mathrm{~W}_{1}^{0}\end{array}\right)$ is approximately $\mathrm{L}^{\infty}$-controllable at a free time. Let us find controls solving the approximate $\mathrm{L}^{\infty}$ controllability problem for this state.

Let $F, F_{m}, \mu, \mu_{m}, m=\overline{1, \infty}$, be the functions from the proof of Theorem 5.9. Then (5.3) holds and

$$
\left\|\mathrm{W}_{0}^{0}-\mathrm{W}_{0}^{m}\right\|_{0}^{0}=\left\|F-F_{m}\right\|_{0}^{0} \leq L_{2 n+2} \sqrt{\frac{\pi}{m}}\left(\frac{q^{2}+4}{q^{2}}\right)^{n+1}, \quad m \geq \frac{6}{q},
$$

where $\mathrm{W}_{0}^{m}=\mathcal{F}^{-1} F_{m}, m=\overline{1, \infty}$. Put $g_{m}^{n}=\sqrt{\frac{\pi}{2}}(-1)^{n} \operatorname{sgn} t \mathcal{F}_{\xi \rightarrow t}^{-1}\left(|\xi| \mu_{m}^{(n)}\left(\sqrt{q^{2}-\xi^{2}}\right) / \sqrt{q^{2}-\xi^{2}}\right), m=\overline{1, \infty}$. With regard to Theorem 5.6 and Remark 5.7, we conclude that $g_{m}^{n} \in N(\widehat{\Psi}) \cap \mathrm{L}^{\infty}(\mathbb{R}), g_{m}^{n}$ is odd and $\Psi g_{m}^{n}=\mathrm{W}_{m}^{0}$, $m=\overline{1, \infty}$. Set $\mathcal{U}_{m, k}^{n}(t)=g_{m}^{n}(t)(H(t+k)-H(t-k)), t \in \mathbb{R}, m, k=\overline{1, \infty}$. Then $\mathcal{U}_{m, k}^{n} \in \mathrm{L}^{\infty}(\mathbb{R}), \mathcal{U}_{m, k}^{n}$ is odd, $m, k=\overline{1, \infty}$, and

$$
\left\|g_{m}^{n}-\mathcal{U}_{m, k}^{n}\right\|_{0}^{0} \leq\left(2 \int_{k}^{\infty}\left|g_{m}^{n}(t)\right|^{2} \mathrm{~d} t\right)^{1 / 2} \rightarrow 0 \quad \text { as } k \rightarrow \infty .
$$

Taking into account Theorem 4.3: (ii) and Theorem 4.4: (ii), we conclude that

$$
\left\|\mathrm{W}_{0}^{m}-\Psi \mathcal{U}_{m, k}^{n}\right\|_{0}^{0} \leq\left\|g_{m}^{n}-\mathcal{U}_{m, k}^{n}\right\|_{0}^{0}, \quad\left\|0-\widehat{\Psi} \mathcal{U}_{m, k}^{n}\right\|_{0}^{-1} \leq\left\|g_{m}^{n}-\mathcal{U}_{m, k}^{n}\right\|_{0}^{0}, \quad m, k=\overline{1, \infty} .
$$

Estimates (7.1)-(7.3), (A.16) yield

$$
\begin{aligned}
\left\|\mathrm{W}_{0}^{0}-\Psi \mathcal{U}_{m, k}^{n}\right\|_{0}^{0} \leq L_{2 n+2} \sqrt{\frac{\pi}{m}}\left(\frac{q^{2}+4}{q^{2}}\right)^{n+1}+\left(2 \int_{k}^{\infty}\left|g_{m}^{n}(t)\right|^{2} \mathrm{~d} t\right)^{1 / 2}, & m \geq \frac{6}{q}, k \geq 1, \\
\left\|\mathrm{~W}_{1}^{0}-\widehat{\Psi} \mathcal{U}_{m, k}^{n}\right\|_{0}^{-1} \leq\left(2 \int_{k}^{\infty}\left|g_{m}^{n}(t)\right|^{2} \mathrm{~d} t\right)^{1 / 2}, & m \geq \frac{6}{q}, k \geq 1,
\end{aligned}
$$

where $L_{2 n+2} \leq 2(4(n+1)) ! \sqrt{(8(n+1)) !} 5^{2(n+1)} / \pi$. 
Let $\varepsilon>0$ be fixed. Determine $m_{\varepsilon} \geq \frac{6}{q}$ such that $L_{2 n+2} \sqrt{\frac{\pi}{m}}\left(\frac{q^{2}+4}{q^{2}}\right)^{n+1}<\frac{\varepsilon}{2}$. Then for this $m_{\varepsilon}$ find $k_{\varepsilon} \geq 1$ such that $\left(2 \int_{k_{\varepsilon}}^{\infty}\left|g_{m_{\varepsilon}}^{n}(t)\right|^{2} \mathrm{~d} t\right)^{1 / 2}<\frac{\varepsilon}{2}$. Taking into account (7.4), (7.5), we obtain

$$
\left\|\mathrm{W}_{0}^{0}-\Psi \mathcal{U}_{m_{\varepsilon}, k_{\varepsilon}}^{n}\right\|_{0}^{0}<\varepsilon \text { and }\left\|\mathrm{W}_{1}^{0}-\widehat{\Psi} \mathcal{U}_{m_{\varepsilon}, k_{\varepsilon}}^{n}\right\|_{0}^{-1}<\varepsilon .
$$

Thus, the controls $u_{m, k}^{n}=\mathcal{U}_{m, k} H(t) \in \mathrm{L}^{\infty}(\mathbb{R}), m, k=\overline{1, \infty}$, solve the approximate $\mathrm{L}^{\infty}$-controllability problem at a free time for the state $\mathrm{W}^{0}$.

In the case $n=0$ the state $\mathrm{W}^{0}$ is also $\mathcal{B}^{U}$-controllable at a free time for $U>1$ because

$$
\left\|g_{m}^{0}\right\|_{\mathrm{L}^{\infty}(\mathbb{R})} \leq \int_{q-3 / m}^{q-1 / m} \mu_{m}^{0}(\xi) \mathrm{d} \xi=\int_{-1}^{1} \mu(\xi) \mathrm{d} \xi=1=\|\operatorname{sgn} t\|_{\mathrm{L}^{\infty}(\mathbb{R})} .
$$

With regard to Remark 5.13, we also have $\Psi_{\infty} \operatorname{sgn} t=\mathrm{W}_{0}^{0}$ and $\Psi g_{m}^{0} \rightarrow \mathrm{W}_{0}^{0}$ as $m \rightarrow \infty$.

Example 7.4. Let $q>0, n=\overline{0, \infty}, \mathrm{W}_{0}^{0}=0, \mathrm{~W}_{1}^{0}=|x|^{n} \mathrm{e}^{-q|x|} \operatorname{sgn} x$. Due to Corollary 4.9, the state $\mathrm{W}^{0}=\left(\begin{array}{c}\mathrm{W}_{0}^{0} \\ \mathrm{~W}_{1}^{0}\end{array}\right)$ is approximately $\mathrm{L}^{\infty}$-controllable at a free time. Let us find controls solving the approximate $\mathrm{L}^{\infty}$-controllability problem for this state. Reasoning as in Example 7.3 and using Theorem 5.4 instead of Theorem 5.6, we conclude that

$$
\begin{array}{cl}
\left\|\mathrm{W}_{0}^{0}-\Psi \mathcal{U}_{m, k}^{n}\right\|_{0}^{0} \leq\left(2 \int_{k}^{\infty}\left|g_{m}^{n}(t)\right|^{2} \mathrm{~d} t\right)^{1 / 2}, & m \geq \frac{6}{q}, k \geq 1, \\
\left\|\mathrm{~W}_{1}^{0}-\widehat{\Psi} \mathcal{U}_{m, k}^{n}\right\|_{0}^{-1} \leq L_{2 n+2} \sqrt{\frac{\pi}{m}}\left(\frac{q^{2}+4}{q^{2}}\right)^{n+1}+\left(2 \int_{k}^{\infty}\left|g_{m}^{n}(t)\right|^{2} \mathrm{~d} t\right)^{1 / 2}, & m \geq \frac{6}{q}, k \geq 1,
\end{array}
$$

where $g_{m}^{n}=\sqrt{\frac{\pi}{2}}(-1)^{n+1} i \mathcal{F}_{\xi \rightarrow t}^{-1}\left(\operatorname{sgn} \xi \mu_{m}^{(n)}\left(\sqrt{q^{2}-\xi^{2}}\right) / \sqrt{q^{2}-\xi^{2}}\right), \mathcal{U}_{m, k}^{n}=g_{m}^{n}(H(t+k)-H(t-k)), \mathcal{U}_{m, k}^{n} \in \mathrm{L}^{\infty}(\mathbb{R})$, $\mathcal{U}_{m, k}^{n}$ is odd, $m, k=\overline{1, \infty}, L_{2 n+2} \leq 2(4(n+1)) ! \sqrt{(8(n+1)) !} 5^{2(n+1)} / \pi$. Thus, the controls $u_{m, k}^{n}=\mathcal{U}_{m, k} H(t) \in$ $\mathrm{L}^{\infty}(\mathbb{R}), m, k=\overline{1, \infty}$, solve the approximate $\mathrm{L}^{\infty}$-controllability problem at a free time for the state $\mathrm{W}^{0}$.

Remark 7.5. It is known that $\left\{\frac{1}{\sqrt{2 q l !}} \mathrm{e}^{q x}\left(\frac{\mathrm{d}}{\mathrm{d} x}\right)^{l}\left(x^{l} \mathrm{e}^{-2 q x}\right)\right\}_{l=0}^{\infty}$ is an orthonormal basis in $\mathrm{L}^{\infty}(0,+\infty)$. Put $\nu_{l}(x)=\frac{1}{\sqrt{q}} \sum_{n=0}^{l}\left(\begin{array}{l}l \\ n\end{array}\right) \frac{(-2 q)^{n}}{n !}|x|^{n} \mathrm{e}^{-q|x|} \operatorname{sgn} x, x \in \mathbb{R}, l=\overline{0, \infty}$. Then $\left\{\nu_{l}\right\}_{l=0}^{\infty}$ is an orthonormal basis in $\widetilde{H}_{0}^{0}$. Let $\mathrm{W}_{0}^{0} \in \widetilde{H}_{0}^{0}, \mathrm{~W}_{1}^{0}=0$. Put $\mathrm{W}_{0}^{0}=\sum_{l=0}^{\infty} \omega_{l} \nu_{l}, \mathrm{~W}_{0}^{N}=\sum_{l=0}^{N} \omega_{l} \nu_{l}, \mathrm{~W}_{1}^{0}=\mathrm{W}_{1}^{N}=0, N=\overline{1, \infty}$, where $\omega_{l}=\left\langle\mathrm{W}_{0}^{0}, \nu_{l}\right\rangle$, $l=\overline{0, \infty}$. Then we have $\left\|\mathrm{W}_{0}^{0}-\mathrm{W}_{0}^{N}\right\|_{0}^{0} \rightarrow 0$ and $\left\|\mathrm{W}_{1}^{0}-\mathrm{W}_{1}^{N}\right\|_{0}^{0} \rightarrow 0$ as $N \rightarrow \infty$. Using the result of Example 7.3, we can find controls solving the approximate $\mathrm{L}^{\infty}$-controllability problem at a free time for $\left(\begin{array}{l}\mathrm{W}_{0}^{N} \\ \mathrm{~W}_{1}^{N}\end{array}\right), N=\overline{1, \infty}$. Therefore, we can find controls solving the approximate $\mathrm{L}^{\infty}$-controllability problem for $\left(\begin{array}{l}\mathrm{W}_{0}^{0} \\ \mathrm{~W}_{1}^{0}\end{array}\right)$. In the following example we realize this scheme.

Example 7.6. Let $q>0, \mathrm{~W}_{0}^{0}=x H\left(1-x^{2}\right), \mathrm{W}_{1}^{0}=0$. According to Corollary 3.9 , the state $\mathrm{W}^{0}=\left(\begin{array}{c}\mathrm{W}_{0}^{0} \\ \mathrm{~W}_{1}^{0}\end{array}\right)$ is approximately $\mathrm{L}^{\infty}$-controllable at a given time $T>0$ iff $T>1$ and condition (3.8) holds. With regard to Theorem 3.1: (vi), we have

$$
\Psi_{1} \frac{\mathrm{d}}{\mathrm{d} t}\left(\operatorname{sgn} t \Psi_{1}^{-1} \mathrm{~W}_{0}^{0}\right)=\Psi_{1} \frac{\mathrm{d}}{\mathrm{d} t}\left(|t| I_{0}\left(q \sqrt{1-t^{2}}\right)\right) \neq 0=\mathrm{W}_{1}^{0} .
$$


Therefore, for all $T>0$ the state $\mathrm{W}^{0}$ is not approximately $\mathrm{L}^{\infty}$-controllable at the time $T$. But due to Corollary 4.9, this state is approximately $\mathrm{L}^{\infty}$-controllable at a free time. Taking into account Remark 7.5, we can find controls solving the approximate $\mathrm{L}^{\infty}$-controllability problem for the state $\mathrm{W}^{0}$. We have $\mathrm{W}_{0}^{0}=\sum_{l=0}^{\infty} \omega_{l} \nu_{l}$, where

$$
\begin{aligned}
\omega_{l}=\left\langle\mathrm{W}_{0}^{0}, \nu_{l}\right\rangle & =\frac{2}{\sqrt{q}} \sum_{n=0}^{l}\left(\begin{array}{l}
l \\
n
\end{array}\right) \frac{(-2 q)^{n}}{n !} \int_{0}^{1} x^{n+1} \mathrm{e}^{-q x} \mathrm{~d} x=\frac{2}{\sqrt{q}} \sum_{n=0}^{l}\left(\begin{array}{l}
l \\
n
\end{array}\right) \frac{(2 q)^{n}}{n !}\left(\frac{\mathrm{d}}{\mathrm{d} q}\right)^{n+1}\left(\frac{\mathrm{e}^{-q}-1}{q}\right) \\
& =\frac{2}{q^{5 / 2}} \sum_{n=0}^{l}\left(\begin{array}{l}
l \\
n
\end{array}\right)(-2)^{n}(n+1) \sum_{m=n+2}^{\infty} \frac{q^{m}}{m !} \mathrm{e}^{-q}, \quad l=\overline{0, \infty} .
\end{aligned}
$$

Put $\mathrm{W}_{0}^{N}=\sum_{l=0}^{N} \omega_{l} \nu_{l}, N=\overline{1, \infty}$. Then $\left\|\mathrm{W}_{0}^{0}-\mathrm{W}_{0}^{N}\right\|_{0}^{0} \rightarrow 0$ as $N \rightarrow \infty$. Let $\varepsilon>0$ be fixed. Set $N=\overline{1, \infty}$ such that

We have

$$
\left\|\mathrm{W}_{0}^{0}-\mathrm{W}_{0}^{N}\right\|_{0}^{0}<\frac{\varepsilon}{2}
$$

where

$$
\mathrm{W}_{0}^{N}=\frac{1}{\sqrt{q}} \sum_{l=0}^{N} \omega_{l} \sum_{n=0}^{l}\left(\begin{array}{l}
l \\
n
\end{array}\right) \frac{(-2 q)^{n}}{n !}|x|^{n} \mathrm{e}^{-q|x|} \operatorname{sgn} x=\sum_{n=0}^{N} \Omega_{n}^{N}|x|^{n} \mathrm{e}^{-q|x|} \operatorname{sgn} x
$$

$$
\Omega_{n}^{N}=\frac{(-2 q)^{n}}{\sqrt{q} n !} \sum_{l=n}^{N}\left(\begin{array}{l}
l \\
n
\end{array}\right) \omega_{l}=\frac{(-1)^{n}}{n !} 2^{n+1} q^{n-3} \sum_{l=n}^{N}\left(\begin{array}{l}
l \\
n
\end{array}\right) \sum_{s=0}^{l}\left(\begin{array}{l}
l \\
s
\end{array}\right)(-2)^{s}(s+1) \sum_{m=s+2}^{\infty} \frac{q^{m}}{m !} \mathrm{e}^{-q}, \quad n=\overline{0, N},
$$

here we use (7.9). According to Example 7.3, for each $n=\overline{0, N}$ we can find an odd function $\mathcal{U}_{\varepsilon}^{n} \in \mathrm{L}^{\infty}(\mathbb{R})$ such that $\operatorname{supp} \mathcal{U}_{\varepsilon}^{n}$ is finite, $\left\||x|^{n} \mathrm{e}^{-q|x|} \operatorname{sgn} x-\Psi \mathcal{U}_{\varepsilon}^{n}\right\|_{0}^{0}<\frac{\varepsilon}{2(N+1)\left|\Omega_{n}^{N}\right|}$, and $\left\|0-\widehat{\Psi} \mathcal{U}_{\varepsilon}^{n}\right\|_{0}^{-1}<\frac{\varepsilon}{2(N+1)\left|\Omega_{n}^{N}\right|}$. Hence, for $\mathcal{U}_{\varepsilon}=\sum_{n=0}^{N} \Omega_{n}^{N} \mathfrak{U}_{\varepsilon}^{n}$ we have $\mathcal{U}_{\varepsilon} \in \mathrm{L}^{\infty}(\mathbb{R}), \mathcal{U}_{\varepsilon}$ is odd, $\operatorname{supp} \mathcal{U}_{\varepsilon}$ is finite, $\left\|\mathrm{W}_{0}^{N}-\Psi \mathcal{U}_{\varepsilon}\right\|_{0}^{0}<\frac{\varepsilon}{2}$, and $\left\|0-\widehat{\Psi} \mathcal{U}_{\varepsilon}\right\|_{0}^{-1}<\frac{\varepsilon}{2}$. With regard to (7.10), we get $\left\|\mathrm{W}_{0}^{0}-\Psi \mathcal{U}_{\varepsilon}\right\|_{0}^{0}<\varepsilon$ and $\left\|\mathrm{W}_{1}^{0}-\widehat{\Psi} \mathcal{U}_{\varepsilon}\right\|_{0}^{-1}<\varepsilon$. Thus, the controls $u_{\varepsilon}=\mathcal{U}_{\varepsilon} H(t) \in$ $\mathrm{L}^{\infty}(\mathbb{R}), \varepsilon>0$, solve the approximate $\mathrm{L}^{\infty}$-controllability problem at a free time for the state $\mathrm{W}^{0}$.

Remark 7.7. It is known that $\widetilde{H}_{0}^{-1}$ is the closure of $\widetilde{H}_{0}^{0}$ with respect to the norm $\|\cdot\|_{0}^{-1}[7]$ Chapter 1 . That is why for $\mathrm{W}_{0}^{0}=0, \mathrm{~W}_{1}^{0} \in \widetilde{H}_{0}^{-1}$ we can find a sequence $\left\{\mathrm{W}_{1}^{M}\right\}_{M=1}^{\infty} \subset \widetilde{H}_{0}^{0}$ such that $\left\|\left(\begin{array}{c}\mathrm{W}_{0}^{0} \\ \mathrm{~W}_{1}^{0}\end{array}\right)-\left(\begin{array}{c}\mathrm{W}_{0}^{M} \\ \mathrm{~W}_{1}^{M}\end{array}\right)\right\| \| 0$ as $M \rightarrow \infty$, where $\mathrm{W}_{0}^{M}=\mathrm{W}_{0}^{0}=0$. Moreover, we have $\left\|\left(\begin{array}{c}\mathrm{W}_{0}^{M} \\ \mathrm{~W}_{1}^{M}\end{array}\right)-\left(\begin{array}{c}\mathrm{W}_{0}^{M, N} \\ \mathrm{~W}_{1}^{M, N}\end{array}\right)\right\| \rightarrow 0$ as $N \rightarrow \infty, M=\overline{1, \infty}$, if $\mathrm{W}_{0}^{M, N}=\mathrm{W}_{0}^{M}=0, \mathrm{~W}_{1}^{M}=\sum_{l=0}^{\infty} \omega_{l} \nu_{l}, \mathrm{~W}_{1}^{M, N}=\sum_{l=0}^{N} \omega_{l} \nu_{l}, M, N=\overline{1, \infty}$. Here $\omega_{l}^{M}=\left\langle\mathrm{W}_{1}^{M}, \nu_{l}\right\rangle, l=\overline{0, \infty}$. Then, using the result of Example 7.4, we can find controls solving the approximate $\mathrm{L}^{\infty}$-controllability problem at a free time for $\left(\begin{array}{l}\mathrm{W}_{0}^{M, N} \\ \mathrm{~W}_{1}^{M, N}\end{array}\right)$, therefore, for $\left(\begin{array}{l}\mathrm{W}_{0}^{M} \\ \mathrm{~W}_{1}^{M}\end{array}\right)$ and for $\left(\begin{array}{c}\mathrm{W}_{0}^{0} \\ \mathrm{~W}_{1}^{0}\end{array}\right)$. In the following example we realize this scheme.

Example 7.8. Let $q>0, \mathrm{~W}_{0}^{0}=0, \mathrm{~W}_{1}^{0}=\frac{2}{x}$. Due to Corollary 4.9 , this state $\mathrm{W}^{0}=\left(\begin{array}{c}\mathrm{W}_{0}^{0} \\ \mathrm{~W}_{1}^{0}\end{array}\right)$ is approximately $\mathrm{L}^{\infty}$-controllable at a free time. Taking into account Remark 7.7, we can find controls solving the approximate $\mathrm{L}^{\infty}$-controllability problem for the state $\mathrm{W}^{0}$. Put $\mathrm{V}_{1}^{0}=\mathcal{F} \mathrm{W}_{1}^{0}=-\mathrm{i} \sqrt{\frac{\pi}{2}} \operatorname{sgn} \sigma, \mathrm{V}_{1}^{M}=\mathrm{V}_{1}^{0}(H(\sigma+M)-H(\sigma-M))$, $\mathrm{W}_{1}^{M}=\mathcal{F}^{-1} \mathrm{~V}_{1}^{M}, \mathrm{~W}_{0}^{M}=0, M=\overline{1, \infty}$. Evidently, $\mathrm{V}_{1}^{M} \in \widetilde{H}_{0}^{0}$, hence, $\mathrm{W}_{1}^{M} \in \widetilde{H}_{0}^{0}$. Then $\left\|\mathrm{W}_{1}^{0}-\mathrm{W}_{1}^{M}\right\|_{0}^{-1}=$ $\left\|\mathrm{V}_{1}^{0}-\mathrm{V}_{1}^{M}\right\|_{-1}^{0}=\sqrt{\pi}\left(\int_{M}^{\infty} \frac{\mathrm{d} \sigma}{1+\sigma^{2}}\right)^{1 / 2}=\sqrt{\pi \operatorname{arccot} M}$. Let $\varepsilon>0$ be fixed. Set $M>0$ such that $\sqrt{\pi \operatorname{arccot} M}<\frac{\varepsilon}{3}$. 
Then

$$
\left\|\left(\begin{array}{c}
\mathrm{W}_{0}^{0} \\
\mathrm{~W}_{1}^{0}
\end{array}\right)-\left(\begin{array}{c}
\mathrm{W}_{0}^{M} \\
\mathrm{~W}_{1}^{M}
\end{array}\right)\right\|<\frac{\varepsilon}{3}
$$

We have $\mathrm{W}_{1}^{M}=\sum_{l=0}^{\infty} \omega_{l}^{M} \nu_{l}$, where

$$
\begin{aligned}
\omega_{l}^{M} & =\left\langle\mathrm{W}_{1}^{M}, \nu_{l}\right\rangle=\frac{1}{\sqrt{q}} \sum_{n=0}^{l}\left(\begin{array}{l}
l \\
n
\end{array}\right) \frac{(-2 q)^{n}}{n !} \int_{-\infty}^{\infty} \mathrm{W}_{1}^{M}(x)|x|^{n} \mathrm{e}^{-q|x|} \operatorname{sgn} x \mathrm{~d} x \\
& =\sqrt{\frac{2}{\pi}} \frac{1}{\sqrt{q}} \sum_{n=0}^{l}\left(\begin{array}{l}
l \\
n
\end{array}\right) \frac{(2 q)^{n}}{n !}\left(\frac{\mathrm{d}}{\mathrm{d} q}\right)^{n} \int_{-\infty}^{\infty} \mathrm{V}_{1}^{M}(x) \frac{-\mathrm{i} \sigma}{\sigma^{2}+q^{2}}=\frac{-1}{\sqrt{q}} \sum_{n=0}^{l}\left(\begin{array}{l}
l \\
n
\end{array}\right) \frac{(2 q)^{n}}{n !}\left(\frac{\mathrm{d}}{\mathrm{d} q}\right)^{n} \ln \frac{M^{2}+q^{2}}{q^{2}} \\
& =\frac{-2}{\sqrt{q}}\left(\ln \frac{M^{2}+q^{2}}{q^{2}}+\sum_{n=1}^{l}\left(\begin{array}{l}
l \\
n
\end{array}\right) \frac{\left.(-2)^{(} n-1\right)}{q n}+\sum_{n=1}^{l}\left(\begin{array}{l}
l \\
n
\end{array}\right) \frac{\left.(-2 q)^{(} n-1\right)}{q n\left(q^{2}+M^{2}\right)^{n}} \sum_{0 \leq 2 k \leq n}\left(\begin{array}{c}
n \\
2 k
\end{array}\right)\left(-M^{2}\right)^{k} q^{n-2 k}\right) .
\end{aligned}
$$

Put $\mathrm{W}_{1}^{M, N}=\sum_{l=0}^{N} \omega_{l} \nu_{l}, N=\overline{1, \infty}$. Then $\left\|\mathrm{W}_{1}^{M}-\mathrm{W}_{1}^{M, N}\right\|_{0}^{0} \rightarrow 0$ as $N \rightarrow \infty$. Set $N=\overline{1, \infty}$ such that

$$
\left\|\mathrm{W}_{1}^{M}-\mathrm{W}_{1}^{M, N}\right\|_{0}^{0}<\frac{\varepsilon}{3}
$$

We have

$$
\mathrm{W}_{1}^{M, N}=\frac{1}{\sqrt{q}} \sum_{l=0}^{N} \omega_{l}^{M} \sum_{n=0}^{l}\left(\begin{array}{l}
l \\
n
\end{array}\right) \frac{(-2 q)^{n}}{n !}|x|^{n} \mathrm{e}^{-q|x|} \operatorname{sgn} x=\sum_{n=0}^{N} \Omega_{n}^{M, N}|x|^{n} \mathrm{e}^{-q|x|} \operatorname{sgn} x
$$

where

$$
\begin{aligned}
\Omega_{n}^{M, N}=\frac{(-2 q)^{n}}{\sqrt{q} n !} & \sum_{l=n}^{N}\left(\begin{array}{l}
l \\
n
\end{array}\right) \omega_{l}^{M}=\frac{(-1)^{n}}{n !} 2^{n+1} q^{n-1} \sum_{l=n}^{N}\left(\begin{array}{l}
l \\
n
\end{array}\right)\left(\ln \frac{M^{2}+q^{2}}{q^{2}}+\sum_{n=1}^{l}\left(\begin{array}{l}
l \\
n
\end{array}\right) \frac{\left.(-2)^{(} n-1\right)}{q n}\right. \\
& \left.+\sum_{n=1}^{l}\left(\begin{array}{l}
l \\
n
\end{array}\right) \frac{(-2 q)^{(n-1)}}{q n\left(q^{2}+M^{2}\right)^{n}} \sum_{0 \leq 2 k \leq n}\left(\begin{array}{c}
n \\
2 k
\end{array}\right)\left(-M^{2}\right)^{k} q^{n-2 k}\right), \quad n=\overline{0, N},
\end{aligned}
$$

here we use (7.12). According to Example 7.4, for each $n=\overline{0, N}$ we can find an odd function $\mathcal{U}_{\varepsilon}^{n} \in \mathrm{L}^{\infty}(\mathbb{R})$ such that $\operatorname{supp} \mathcal{U}_{\varepsilon}^{n}$ is finite, $\mathcal{U}_{\varepsilon}^{n}$ is odd, $\left\|0-\Psi \mathcal{U}_{\varepsilon}^{n}\right\|_{0}^{0}<\frac{\varepsilon}{\left.3(N+1) \mid \Omega_{n}^{N}\right\rceil}$, and $\left\||x|^{n} \mathrm{e}^{-q|x|} \operatorname{sgn} x-\widehat{\Psi} \mathcal{U}_{\varepsilon}^{n}\right\|_{0}^{-1}<\frac{\varepsilon}{3(N+1)\left|\Omega_{n}^{N}\right|}$. Hence, for $\mathcal{U}_{\varepsilon}=\sum_{n=0}^{N} \Omega_{n}^{M, N} \mathcal{U}_{\varepsilon}^{n}$ we have $\mathcal{U}_{\varepsilon} \in \mathrm{L}^{\infty}(\mathbb{R})$, supp $\mathcal{U}_{\varepsilon}$ is finite, $\mathcal{U}_{\varepsilon}$ is odd, $\left\|0-\Psi \mathcal{U}_{\varepsilon}\right\|_{0}^{0}<\frac{\varepsilon}{3}$, and $\left\|\mathrm{W}_{1}^{M, N}-\widehat{\Psi} \mathcal{U}_{\varepsilon}\right\|_{0}^{-1}<\frac{\varepsilon}{3}$. With regard to (7.11), (7.13), we get $\left\|\mathrm{W}_{0}^{0}-\Psi \mathcal{U}_{\varepsilon}\right\|_{0}^{0}<\varepsilon$ and $\left\|\mathrm{W}_{1}^{0}-\widehat{\Psi} \mathcal{U}_{\varepsilon}\right\|_{0}^{-1}<\varepsilon$. Thus, the controls $u_{\varepsilon}=\mathcal{U}_{\varepsilon} H(t) \in \mathrm{L}^{\infty}(\mathbb{R}), \varepsilon>0$, solve the approximate $\mathrm{L}^{\infty}$-controllability problem at a free time for the state $\mathrm{W}^{0}$.

Example 7.9. Let $q>0, \mathrm{~W}_{0}^{0}=\mathrm{e}^{-q|x|} \operatorname{sgn} x, \mathrm{~W}_{1}^{0}=0$. In Example 7.3 we have proved that the state $\mathrm{W}^{0}=$ $\left(\begin{array}{l}W_{0}^{0} \\ W_{1}^{0}\end{array}\right)$ was approximately $\mathrm{L}^{\infty}$-controllable at a free time. Controls solving the approximate $\mathrm{L}^{\infty}$-controllability problem has been also constructed for this state. But these controls are of a rather complicated form. Due to Remark 5.13, we have $\Psi_{\infty}(\operatorname{sgn} t)=\mathrm{W}_{0}^{0}$. We may suppose that for the state $\mathrm{W}^{0}$ controls solving the 
approximate $\mathrm{L}^{\infty}$-controllability problem at a free time can be constructed in the "natural" form $u_{m}(t)=$ $\operatorname{sgn} t(H(t)-H(t-m)), t \in \mathbb{R}, m=\overline{1, \infty}$. Unfortunately, $\widehat{\Psi} \mathcal{U}_{m}$ does not tend to $\mathrm{W}_{1}^{0}=0$ as $m \rightarrow \infty$ because

$$
\left\|\widehat{\Psi} \mathcal{U}_{m}\right\|_{0}^{-1} \geq \frac{1}{\pi} \sqrt{\frac{q}{1+q^{2}}}, \quad m \geq \frac{3 \pi}{2 q},
$$

where $\mathcal{U}_{m}(t)=u_{m}(t)-u_{m}(-t), m=\overline{1, \infty}$. Therefore, controls $u_{m}, m=\overline{1, \infty}$, do not solve the problem under consideration. Let us prove estimate (7.14). We have

$$
\begin{aligned}
\left(\left\|\widehat{\Psi} \mathcal{U}_{m}\right\|_{0}^{-1}\right)^{2} & =\left(\left\|\mathcal{F} \widehat{\Psi} \mathcal{U}_{m}\right\|_{-1}^{0}\right)^{2}=\frac{4}{\pi} \int_{0}^{\infty}\left|\frac{\sigma \sin \left(m \sqrt{\sigma^{2}+q^{2}}\right)}{\sqrt{\sigma^{2}+q^{2}}}\right|^{2} \frac{\mathrm{d} \sigma}{\sigma^{2}+1} \\
& \geq \frac{4 q^{2}}{\pi\left(q^{2}+1\right)} \int_{q}^{\infty} \sin ^{2}(m \xi) \frac{\sqrt{\xi^{2}-q^{2}}}{\xi^{3}} \mathrm{~d} \xi \geq \frac{q}{\pi\left(q^{2}+1\right)}-\frac{2 m q^{2}}{\pi\left(q^{2}+1\right)}\left|\int_{m q}^{\infty} \cos (2 p) \frac{p-m q}{p^{3}} \mathrm{~d} p\right| .
\end{aligned}
$$

Now estimate the later integral. Determine $n_{m} \in \mathbb{Z}$ such that $\frac{\pi}{4}+\pi n_{m} \leq q<\frac{5 \pi}{4}+\pi n_{m}$. Then

$$
\begin{aligned}
m\left|\int_{m q}^{\infty} \cos (2 p) \frac{p-m q}{p^{3}} \mathrm{~d} p\right| & \leq m \sum_{n=n_{m}}^{\infty} \int_{3 \pi / 4}^{5 \pi / 4} \cos (2 p) \frac{p-m q}{p^{3}} \mathrm{~d} p \leq \frac{m}{\pi^{2}} \sum_{n=n_{m}}^{\infty} \frac{n+\frac{5}{4}+\frac{q m}{\pi}}{\left(n+\frac{3}{4}\right)^{3}} \\
& \leq \frac{m}{\pi^{2}} \int_{n_{m}}^{\infty} \frac{x+\frac{5}{4}+\frac{q m}{\pi}}{\left(x+\frac{3}{4}\right)^{3}} \mathrm{~d} x \leq \frac{q m^{2}-\frac{3 \pi}{2}}{2 \pi\left(q m-\frac{\pi}{2}\right)} \rightarrow \frac{1}{2 \pi q} \quad \text { as } m \rightarrow \infty .
\end{aligned}
$$

Hence, $m \int_{m q}^{\infty}|\cos (2 p)| \frac{p-m q}{p^{3}} \mathrm{~d} p \leq \frac{1}{\pi q}, m \geq \frac{3 \pi}{2 q}$. With regard to (7.15), we conclude that estimate (7.15) is valid.

In the following examples we use Theorem 6.3 to investigate the approximate $\mathcal{B}^{U}$-controllability problem at a free time.

Example 7.10. Let $q>0, \mathrm{~W}_{0}^{0}=\operatorname{sgn} x \frac{1-(1+q|x|) \mathrm{e}^{-q|x|}}{x^{2}}, \mathrm{~W}_{1}^{0}=0$. Then $\left(\mathrm{W}_{0}^{0}\right)_{\mathbb{1}}=\frac{\mathrm{d}}{\mathrm{d} y}\left(\frac{1-\mathrm{e}^{-\mathrm{i} q y}}{y}\right),\left(\mathrm{W}_{1}^{0}\right)_{\mathbb{1}}=0$, $\Phi_{0}=-\frac{1}{2} \mathcal{F}^{-1}\left(\mathrm{~W}_{0}^{0}\right)_{(1)}=\sqrt{\frac{\pi}{2}} \mathrm{i} \xi(H(\xi)-H(\xi-q)), \Phi_{1}=0$. Since $\Phi_{0}$ and $\Phi_{1}$ satisfy the conditions of the Theorems 5.6 and 5.4 respectively, we have $\mathrm{W}_{0}^{0} \in \Psi(N(\widehat{\Psi}))$ and $\mathrm{W}_{1}^{0} \in \widehat{\Psi}(N(\Psi))$. Due to Theorem 6.3, the state $\mathrm{W}^{0}=\left(\begin{array}{c}\mathrm{W}_{0}^{0} \\ \mathrm{~W}_{1}^{0}\end{array}\right)$ is approximately $\mathcal{B}^{U}$-controllable at a free time for

$$
U \geq U^{*}=\left\|\Phi_{0}\right\|_{L^{1}(\mathbb{R})}=\frac{q^{2}}{2}
$$

Now let us find controls solving the approximate $\mathcal{B}^{U}$-controllability problem at a free time for $\mathrm{W}^{0}$. To this aid, we use the scheme from the proof of Theorem 6.3. Put $\mathcal{U}=g_{0}=\operatorname{sgn} t \mathcal{F}_{\xi \rightarrow t}^{-1}\left(\mathrm{i}|\xi| \frac{\Phi_{0}\left(\sqrt{q^{2}-\xi^{2}}\right)}{\sqrt{q^{2}-\xi^{2}}}\right)=$ $\operatorname{sgn} t\left(\frac{1-\cos (q t)}{t^{2}}-q \frac{\sin (q t)}{t}\right)$. Then the controls $u_{k}(t)=U(t)(H(t)-H(t-k)), t \in \mathbb{R}, k=\overline{1, \infty}$, solve the approximate $\mathcal{B}^{U}$-controllability problem at a free time for $\mathrm{W}^{0}$. Moreover, we have

$$
\frac{q^{2}}{2}=\mathcal{U}_{k}(+0) \leq\left\|\mathcal{U}_{k}\right\|_{L^{\infty}(\mathbb{R})} \leq\|\mathcal{U}\|_{L^{\infty}(\mathbb{R})}=\left\|g_{0}\right\|_{L^{\infty}(\mathbb{R})} \leq \int_{0}^{q} \xi \mathrm{d} \xi=\frac{q^{2}}{2} .
$$

Therefore, estimate (7.16) is sharp for this $\mathrm{W}^{0}$. 
Example 7.11. Let $q>0, \mathrm{~W}_{0}^{0}=0, \mathrm{~W}_{1}^{0}=\operatorname{sgn} x \int_{0}^{q} \operatorname{sgn}\left(\xi-\frac{\sqrt{3} q}{2}\right) \sqrt{q^{2}-\xi^{2}} \mathrm{e}^{-\xi|x|} \mathrm{d} \xi$. Then $\left(\mathrm{W}_{0}^{0}\right)_{\mathbb{1}}=0$, $\left(\mathrm{W}_{1}^{0}\right)_{\mathbb{1}}=\int_{0}^{q} \operatorname{sgn}\left(\xi-\frac{\sqrt{3} q}{2}\right) \sqrt{q^{2}-\xi^{2}} \mathrm{e}^{-\mathrm{i} \xi y} \mathrm{~d} \xi, \Phi_{0}=0, \Phi_{1}=\mathrm{i} \sqrt{\frac{\pi}{2}} \operatorname{sgn}\left(\xi-\frac{\sqrt{3} q}{2}\right) \sqrt{q^{2}-\xi^{2}}(H(\xi)-H(\xi-q))$. Since $\Phi_{0}$ and $\Phi_{1}$ satisfy the conditions of the Theorems 5.6 and 5.4 respectively, we have $\mathrm{W}_{0}^{0} \in \Psi(N(\widehat{\Psi}))$ and $\mathrm{W}_{1}^{0} \in \widehat{\Psi}(N(\Psi))$. Due to Theorem 6.3 the state $\mathrm{W}^{0}=\left(\begin{array}{c}\mathrm{W}_{0}^{0} \\ \mathrm{~W}_{1}^{0}\end{array}\right)$ is approximately $\mathcal{B}^{U}$-controllable at a free time for

$$
U \geq U^{*}=\left\|\Phi_{1}\right\|_{L^{1}(\mathbb{R})}=q
$$

Moreover, the controls $u_{k}(t)=\mathcal{U}(t)(H(t)-H(t-k)), t \in \mathbb{R}, k=\overline{1, \infty}$, solve the approximate $\mathcal{B}^{U}$-controllability problem at a free time for $\mathrm{W}^{0}$. Here $\mathcal{U}=g_{1}=\mathcal{F}_{\xi \rightarrow t}^{-1}\left(\operatorname{sgn} \xi \frac{\Phi_{1}\left(\sqrt{q^{2}-\xi^{2}}\right)}{\sqrt{q^{2}-\xi^{2}}}\right)=2 \frac{\sin (q t / 2)}{t}(1-\cos (q t))$. We have

$$
U_{*}=\|\mathcal{U}\|_{L^{\infty}(\mathbb{R})}=\left\|g_{1}\right\|_{L^{\infty}(\mathbb{R})}=\sup _{t>0}\left|2 \frac{\sin (q t / 2)}{t}(1-\cos (q t))\right|<q=U^{*} .
$$

Therefore, estimate (7.17) is not sharp for this $\mathrm{W}^{0}$.

\section{Appendix A}

Lemma A.12. Let $q>0$. Then

$$
|\|E(\cdot, t) * f\|| \leq 2 \frac{1+q^{2}}{q}\|f\| \mid, \quad t \in \mathbb{R}, \quad f \in H_{0}^{0} \times H_{0}^{-1}
$$

Proof. With regard to [4], we have

$$
(\mathcal{F} E(\cdot, t))(\sigma)=\left(\begin{array}{cc}
\partial / \partial t & 1 \\
(\partial / \partial t)^{2} & \partial / \partial t
\end{array}\right) \frac{\sin \left(t \sqrt{\sigma^{2}+q^{2}}\right)}{\sqrt{\sigma^{2}+q^{2}}}, \quad t \in \mathbb{R}, \sigma \in \mathbb{R} .
$$

Put $F=\mathcal{F} f$. Obviously, $F=\left(\begin{array}{c}F_{0} \\ F_{1}\end{array}\right) \in H_{0}^{0} \times H_{-1}^{0}$.Hence,

$$
\begin{aligned}
& \|E(\cdot, t) * f\| \| \\
& \leq \sqrt{2}\left(\left(\left\|F_{0}\right\|_{0}^{0}\right)^{2}+\left(\left\|\frac{\sin \left(t \sqrt{\sigma^{2}+q^{2}}\right)}{\sqrt{\sigma^{2}+q^{2}}} F_{1}\right\|_{0}^{0}\right)^{2}+\left(\left\|\sqrt{\sigma^{2}+q^{2}} F_{0}\right\|_{-1}^{0}\right)^{2}+\left(\left\|F_{1}\right\|_{-1}^{0}\right)^{2}\right)^{1 / 2}=2 \frac{1+q^{2}}{q}\|f\| \| .
\end{aligned}
$$

The lemma is proved.

Lemma A.13. Let $q>0$. Then $R(\Psi)=\left\{f \in \widetilde{H}_{0}^{0} \mid \exists \tilde{f} \in H_{0}^{1 / 2} \mathcal{F} f=\sqrt{|\sigma|} \mathcal{F} \tilde{f}\right\}$ and $\overline{R(\Psi)}=\widetilde{H}_{0}^{0}$.

Proof. With regard to the definition of $\Psi$, we conclude that $R(\Psi) \subset\left\{f \in \widetilde{H}_{0}^{0} \mid \exists \tilde{f} \in H_{0}^{1 / 2} \mathcal{F} f=\sqrt{|\sigma|} \mathcal{F} \tilde{f}\right.$. Assume that for $f \in \widetilde{H}_{0}^{0}$ there exists $\tilde{f} \in H_{0}^{1 / 2}$ such that $\mathcal{F} f=\sqrt{|\sigma|} \mathcal{F} \widetilde{f}$. Put $g=\mathcal{F}_{\mu \rightarrow t}^{-1}\left(\frac{\mu H\left(\mu^{2}-q^{2}\right)}{\sqrt[4]{\mu^{2}-q^{2}}}(\mathcal{F} \widetilde{f})\left(\sqrt{\mu^{2}-q^{2}}\right)\right)$. 
We have $\|g\|_{0}^{0} \leq \sqrt[4]{1+q^{2}}\|\widetilde{f}\|_{0}^{1 / 2}$. Hence, $g \in D(\Psi)$. Moreover, $\Psi g=\mathcal{F}^{-1}(\sqrt{|\sigma|} \mathcal{F} \widetilde{f})=f$. Therefore, $R(\Psi)=\left\{f \in \widetilde{H}_{0}^{0} \mid \exists \tilde{f} \in H_{0}^{1 / 2} \mathcal{F} f=\sqrt{|\sigma|} \mathcal{F} \tilde{f}\right\}$. Let $f \in \widetilde{H}_{0}^{0} \backslash R(\Psi)$. Put $f_{n}(x)=H\left(n^{2}-x^{2}\right) f(x)$. Evidently, $\left\|f-f_{n}\right\|_{0}^{0} \rightarrow 0$ as $n \rightarrow \infty$. Due to the Paley-Wiener Theorem, we conclude that $\mathcal{F} f_{n}$ can be extended to an entire function hence $\frac{1}{\sqrt{|\sigma|}} \mathcal{F} f_{n} \in H_{1 / 2}^{0}$. Therefore, $f_{n} \in R(\Psi)$. Thus, $f \in \overline{R(\Psi)}$. The lemma is proved.

Lemma A.14. Let $q>0$. Then

$$
\Psi^{+} f=f(t)-\int_{|t|}^{\infty} \psi_{\infty}(t, x) f(x) \mathrm{d} x+\int_{0}^{|t|} \psi_{0}(t, x) f(x) \mathrm{d} x, \quad f \in R(\Psi),
$$

where $\psi_{0}, \psi_{\infty}$ are defined in Section 4.

Proof. Let $f \in R(\Psi)$. According to (4.3), we obtain

$$
\Psi^{+} f=-\frac{1}{2} \frac{\mathrm{d}}{\mathrm{d} t} \int_{-\infty}^{\infty} f(x)\left(\frac{2}{\pi} \int_{q}^{\infty} \cos (t \mu) \frac{\sin \left(x \sqrt{\mu^{2}-q^{2}}\right)}{\sqrt{\mu^{2}-q^{2}}} \mathrm{~d} \mu\right) \mathrm{d} x .
$$

Let us study the internal integral. We have

$$
\begin{aligned}
I=\frac{2}{\pi} \int_{q}^{\infty} \cos (t \mu) \frac{\sin \left(x \sqrt{\mu^{2}-q^{2}}\right)}{\sqrt{\mu^{2}-q^{2}}} \mathrm{~d} \mu= & \frac{1}{\pi} \int_{0}^{\infty} \frac{\sin \left(x \sigma+t \sqrt{\sigma^{2}+q^{2}}\right)}{\sqrt{\sigma^{2}+q^{2}}} \mathrm{~d} \sigma \\
& +\frac{1}{\pi} \int_{0}^{\infty} \frac{\sin \left(x \sigma-t \sqrt{\sigma^{2}+q^{2}}\right)}{\sqrt{\sigma^{2}+q^{2}}} \mathrm{~d} \sigma .
\end{aligned}
$$

Put $v=\frac{1}{q}\left(x \sigma+t \sqrt{\sigma^{2}+q^{2}}\right)$ in the first integral and $v=\frac{1}{q}\left(x \sigma-t \sqrt{\sigma^{2}+q^{2}}\right)$ in the second one. Then we obtain

$$
I=\frac{2}{\pi} \operatorname{sgn} x\left(H\left(x^{2}-t^{2}\right) \int_{|t|}^{\infty} \frac{\sin (q v) \mathrm{d} v}{\sqrt{v^{2}+x^{2}-t^{2}}}-H\left(t^{2}-x^{2}\right) \int_{\sqrt{t^{2}-x^{2}}}^{|t|} \frac{\sin (q v) \mathrm{d} v}{\sqrt{v^{2}+x^{2}-t^{2}}}\right) .
$$

By substituting (A.4) in (A.3) and integrating by parts, we obtain (A.2). The lemma is proved.

Lemma A.15. Let $\alpha>0$. Then

$$
\left(\Psi_{\alpha} \frac{\mathrm{d}}{\mathrm{d} t}\left(\operatorname{sgn} t \Psi_{\alpha}^{-1} f\right)\right)(x)=(\operatorname{sgn} x f(x))^{\prime}+q \operatorname{sgn} x \int_{|x|}^{\infty} f(\xi) \frac{I_{1}(q(|x|-\xi))}{|x|-\xi} \mathrm{d} \xi, \quad f \in R\left(\Psi_{\alpha}\right) .
$$

Proof. Let $f \in \mathcal{D}(-\alpha, \alpha)=\left\{\varphi \in C^{\infty}(\mathbb{R}): \operatorname{supp} \varphi \subset(-\alpha, \alpha)\right.$ be odd $\}, h=\Psi_{\alpha} \frac{\mathrm{d}}{\mathrm{d} t}\left(\operatorname{sgn} t \Psi_{\alpha}^{-1} f\right)$. We have

$$
G=\frac{1}{\mathrm{i} \xi} \mathcal{F}_{t \rightarrow \xi}\left(\frac{\mathrm{d}}{\mathrm{d} t}\left(\operatorname{sgn} t\left(\Psi_{\alpha}^{-1} f\right)(t)\right)\right)=\frac{-\mathrm{i}}{\pi} \mathcal{P} \frac{1}{\xi} * \frac{\xi(\mathcal{F} f)\left(\sqrt{\xi^{2}-q^{2}}\right)}{\sqrt{\xi^{2}-q^{2}}}=\frac{-2 i}{\pi} \mathrm{V} \cdot \mathrm{p} \cdot \int_{0}^{\infty} \frac{\left(\mathcal{F} \Delta_{q} f\right)\left(\sqrt{\mu^{2}-q^{2}}\right)}{\left(\xi^{2}-\mu^{2}\right) \sqrt{\mu^{2}-q^{2}}} \mathrm{~d} \mu,
$$


where $\Delta_{q}=-\left(\frac{\mathrm{d}}{\mathrm{d} x}\right)^{2}-q^{2}$. Therefore,

$$
\begin{aligned}
& h=\frac{\mathrm{d}}{\mathrm{d} x} \mathcal{F}_{\sigma \rightarrow x}^{-1} G\left(\sqrt{\sigma^{2}+q^{2}}\right)=-\mathrm{i} \sqrt{\frac{2}{\pi}} \frac{\mathrm{d}}{\mathrm{d} x}\left(\int_{0}^{q} \frac{\mathrm{e}^{-|x|} \sqrt{q^{2}-\mu^{2}}}{\sqrt{q^{2}-\mu^{2}}} \frac{\left(\mathcal{F} \Delta_{q} f\right)\left(\sqrt{\mu^{2}-q^{2}}\right)}{\sqrt{\mu^{2}-q^{2}}} \mathrm{~d} \mu\right. \\
& \left.-\int_{q}^{\infty} \frac{\sin \left(|x| \sqrt{\mu^{2}-q^{2}}\right)}{\sqrt{\mu^{2}-q^{2}}} \frac{\left(\mathcal{F} \Delta_{q} f\right)\left(\sqrt{\mu^{2}-q^{2}}\right)}{\sqrt{\mu^{2}-q^{2}}} \mathrm{~d} \mu\right) \\
& =\sqrt{\frac{2}{\pi}} \operatorname{sgn} x\left(\int_{0}^{\infty} \Delta_{q} f(\nu) \int_{0}^{q} \frac{\mathrm{e}^{-|x| \sqrt{q^{2}-\mu^{2}}} \sinh \left(\nu \sqrt{q^{2}-\mu^{2}}\right)}{\sqrt{q^{2}-\mu^{2}}} \mathrm{~d} \mu\right. \\
& \left.+\int_{0}^{\infty} \Delta_{q} f(\nu) \int_{q}^{\infty} \frac{\cos \left(x \sqrt{\mu^{2}-q^{2}}\right) \sin \left(\nu \sqrt{\mu^{2}-q^{2}}\right)}{\sqrt{\mu^{2}-q^{2}}} \mathrm{~d} \mu\right) \\
& =\frac{1}{\pi} \operatorname{sgn} x\left(\int_{-\infty}^{\infty} \Delta_{q} f(\nu) \int_{0}^{q} \frac{\mathrm{e}^{-(|x|-\nu) \mu}}{\sqrt{q^{2}-\mu^{2}}} \mathrm{~d} \mu \mathrm{d} \nu-\int_{-\infty}^{\infty} \Delta_{q} f(\nu) \int_{q}^{\infty} \frac{\sin \left((x-\nu) \sqrt{\mu^{2}-q^{2}}\right)}{\sqrt{\mu^{2}-q^{2}}} \mathrm{~d} \mu \mathrm{d} \nu\right)
\end{aligned}
$$

According to (4.4) and (4.5), we get

$$
\frac{2}{\pi} \int_{0}^{q} \frac{\mathrm{e}^{-\xi \mu}}{\sqrt{q^{2}-\mu^{2}}} \mathrm{~d} \mu=I_{0}(q \xi)-L_{0}(q \xi), \quad \xi \in \mathbb{R}
$$

We also have

$$
\begin{aligned}
\mathcal{F}\left(\operatorname{sgn} \xi\left(I_{0}(q|\xi|)-L_{0}(q|\xi|)\right)\right) & =\frac{2}{\pi} \mathcal{F}\left(\operatorname{sgn} \xi \int_{0}^{q} \frac{\mathrm{e}^{-u|\xi|}}{\sqrt{q^{2}-u^{2}}} \mathrm{~d} u\right) \\
& =\left(\frac{2}{\pi}\right)^{3 / 2} \int_{0}^{q} \frac{(-\mathrm{i} \sigma)}{\left(\sigma^{2}+u^{2}\right) \sqrt{q^{2}-u^{2}}} \mathrm{~d} u=\sqrt{\frac{2}{\pi}} \frac{(-\mathrm{i} \operatorname{sgn} \sigma)}{\sqrt{\sigma^{2}+q^{2}}} .
\end{aligned}
$$

Therefore,

$$
\frac{2}{\pi} \int_{q}^{\infty} \frac{\sin \left(\xi \sqrt{\mu^{2}-q^{2}}\right)}{\sqrt{\mu^{2}-q^{2}}}=\frac{2}{\pi} \int_{0}^{\infty} \frac{\sin \sigma \xi}{\sqrt{\sigma^{2}+q^{2}}} \mathrm{~d} \sigma=-\sqrt{\frac{2}{\pi}} \mathcal{F}^{-1} \frac{\mathrm{i} \operatorname{sgn} \sigma}{\sqrt{\sigma^{2}+q^{2}}}=\operatorname{sgn} \xi\left(I_{0}(q|\xi|)-L_{0}(q|\xi|)\right) .
$$

Comparing (A.6)-(A.8), we obtain

$$
\begin{aligned}
h & =\frac{1}{2} \operatorname{sgn} x\left(\left(\Delta_{q} f\right) *\left(I_{0}(q x)-L_{0}(q x)\right)(|x|)-\left(\left(\Delta_{q} f\right) *\left(\operatorname{sgn} x\left(I_{0}(q x)-L_{0}(q|x|)\right)\right)\right)(x)\right) \\
& =\operatorname{sgn} x\left(f *\left(\Delta_{q}\left(H(-x) I_{0}(q x)\right)\right)\right)(|x|)=\operatorname{sgn} x\left(f^{\prime}(x)+f *\left(H(-x) \Delta_{q} I_{0}(q x)\right)\right) .
\end{aligned}
$$

Since $\Delta_{q} I_{0}(q \xi)=\left(q^{2} / 2\right)\left(I_{0}(q \xi)-I_{2}(q \xi)\right)=q I_{1}(q \xi) / \xi$, we conclude that (A.5) is true for $f \in R\left(\Psi_{\alpha}\right) \cap \mathcal{D}(-\alpha, \alpha)$. Let us extend this formula on $R\left(\Psi_{\alpha}\right)$. Let $f \in R\left(\Psi_{\alpha}\right)$. Since $\mathcal{D}(-\alpha, \alpha)$ is dense in $L^{2}(-\alpha, \alpha)$, we can find a 
sequence $\left\{f_{n}\right\}_{n \in \mathbb{N}} \subset \mathcal{D}(-\alpha, \alpha)$ such that $\left\|f-f_{n}\right\|_{0}^{0} \rightarrow \infty$ as $n \rightarrow \infty$. We have

$$
\begin{aligned}
& \left|\int_{|x|}^{\infty} \frac{I_{1}(q(|x|-\xi))}{|x|-\xi}\left(f(\xi)-f_{n}(\xi)\right) \mathrm{d} \xi\right| \\
& \quad \leq \mathrm{e}^{-q|x|}\left(\int_{0}^{\infty} \mathrm{e}^{2 q \xi}\left|f(\xi)-f_{n}(\xi)\right|^{2} \mathrm{~d} \xi\right)^{1 / 2}\left(\int_{0}^{\infty} \mathrm{e}^{-2 q \xi}\left|\frac{I_{1}(q \xi)}{\xi}\right|^{2} \mathrm{~d} \xi\right)^{1 / 2}=2 q K \mathrm{e}^{-q|x|}\left\|f-f_{n}\right\|_{0}^{0}, \quad x \in \mathbb{R},
\end{aligned}
$$

where $K=\frac{\mathrm{e}^{q \alpha}}{2 q}\left(\int_{0}^{\infty} \mathrm{e}^{-2 q \xi}\left|\frac{I_{1}(q \xi)}{\xi}\right|^{2} \mathrm{~d} \xi\right)^{1 / 2}$. Therefore,

$$
\left\|\int_{|x|}^{\infty} \frac{I_{1}(q(|x|-\xi))}{|x|-\xi}\left(f(\xi)-f_{n}(\xi)\right) \mathrm{d} \xi\right\|_{0}^{0} \leq K\left\|f-f_{n}\right\|_{0}^{0} .
$$

Since $\left\|\left(\operatorname{sgn} x\left(f-f_{n}\right)\right)^{\prime}\right\|_{0}^{-1} \leq\left\|f-f_{n}\right\|_{0}^{0}$, then (A.9) yields (A.5) for $f \in R\left(\Psi_{\alpha}\right)$. The lema is proved.

Lemma A.16. Let $f \in \widetilde{H}_{0}^{0}$. Then $\widehat{\Psi} \Psi^{+} f \in \widetilde{H}_{0}^{-1}$,

$$
\begin{aligned}
\widehat{\Psi} \Psi^{+} f & =(\operatorname{sgn} x f)^{\prime}+\frac{q}{2} \operatorname{sgn} x\left(f(x) * \frac{I_{1}(q|x|)-L_{1}(q x)}{x}\right)=\operatorname{sgn} x \mathcal{F}_{\sigma \rightarrow x}^{-1}\left(i \operatorname{sgn} \sigma \sqrt{\sigma^{2}+q^{2}}(\mathcal{F} f)(\sigma)\right) \\
& =(\operatorname{sgn} x f)^{\prime}+q^{2} \operatorname{sgn} x \mathcal{F}_{\sigma \rightarrow x}^{-1}\left(\frac{\mathrm{i} \operatorname{sgn} \sigma(\mathcal{F} f)(\sigma)}{|\sigma|+\sqrt{\sigma^{2}+q^{2}}}\right), \quad f \in R(\Psi),
\end{aligned}
$$

and $\left\|\widehat{\Psi} \Psi^{+}\right\| \leq \sqrt{1+q^{2}}$. Moreover, the operator $\widehat{\Psi} \Psi^{+}$can be continued on $\widetilde{H}_{0}^{0}$ with the same norm by (A.10). Proof. Let $f \in \mathcal{D}=\bigcup_{\alpha>0} \mathcal{D}(-\alpha, \alpha)$ be odd. With regard to (4.3), (A.6), and (A.8), we conclude that

$$
\widehat{\Psi} \Psi^{+} f=\mathrm{i} \sqrt{\frac{2}{\pi}} \frac{\mathrm{d}}{\mathrm{d} x}\left(\int_{q}^{\infty} \frac{\sin \left(|x| \sqrt{\mu^{2}-q^{2}}\right)}{\sqrt{\mu^{2}-q^{2}}} \frac{\left(\mathcal{F} \Delta_{q} f\right)\left(\sqrt{\mu^{2}-q^{2}}\right)}{\sqrt{\mu^{2}-q^{2}}} \mathrm{~d} \mu\right)
$$

Setting $\sigma=\sqrt{\mu^{2}-q^{2}}$, we obtain

$$
\begin{aligned}
\widehat{\Psi} \Psi^{+} f & =\operatorname{sgn} x \mathcal{F}_{\sigma \rightarrow x}^{-1}\left(\frac{\mathrm{i} \operatorname{sgn} \sigma}{\sqrt{\sigma^{2}+q^{2}}}\left(\mathcal{F} \Delta_{q} f\right)(\sigma)\right) \\
& =\mathcal{F}_{\sigma \rightarrow x}^{-1}\left(i \operatorname{sgn} \sigma \sqrt{\sigma^{2}+q^{2}}(\mathcal{F} f)(\sigma)\right)=(\operatorname{sgn} x f)^{\prime}+q^{2} \operatorname{sgn} x \mathcal{F}_{\sigma \rightarrow x}^{-1}\left(\frac{i \operatorname{sgn} \sigma(\mathcal{F} f)(\sigma)}{|\sigma|+\sqrt{\sigma^{2}+q^{2}}}\right) .
\end{aligned}
$$

It also follows from (A.11) that

$$
\begin{aligned}
\widehat{\Psi} \Psi^{+} f & =-\frac{1}{2} \operatorname{sgn} x\left(\left(\Delta_{q} f\right) *\left(\operatorname{sgn} x\left(I_{0}(q x)-L_{0}(q|x|)\right)\right)\right)(x)=\operatorname{sgn} x\left(f^{\prime}(x)+f *\left(\operatorname{sgn} x\left(\Delta_{q} I_{0}(q x)\right)-\Delta_{q} L_{0}(q x)\right)\right) \\
& =(\operatorname{sgn} x f(x))^{\prime}+\frac{q}{2} \operatorname{sgn} x\left(f(x) * \frac{I_{1}(q|x|)-L_{1}(q x)}{x}\right) .
\end{aligned}
$$


Thus, (A.10) is true for $f \in \mathcal{D} \cap \widetilde{H}_{0}^{0}$. Let us extend this formula on $\widetilde{H}_{0}^{0}$ and continue the operator $\widehat{\Psi} \Psi^{+}$on $\widetilde{H}_{0}^{0}$. Since $\mathcal{D}$ is dense in $L^{2}(\mathbb{R})$, we can find a sequence $\left\{f_{n}\right\}_{n \in \mathbb{N}} \subset \mathcal{D}$ such that $\left\|f-f_{n}\right\|_{0}^{0} \rightarrow \infty$ as $n \rightarrow \infty$. We have

$$
\left\|\operatorname{sgn} x \mathcal{F}^{-1}\left(\frac{i\left(\mathcal{F}\left(f-f_{n}\right)\right)(\sigma)}{\sigma+\operatorname{sgn} \sigma \sqrt{\sigma^{2}+q^{2}}}\right)\right\|_{0}^{0}=\left\|\frac{\left(\mathcal{F}\left(f-f_{n}\right)\right)(\sigma)}{\sigma+\operatorname{sgn} \sigma \sqrt{\sigma^{2}+q^{2}}}\right\|_{0}^{0} \leq \frac{1}{q}\left\|f-f_{n}\right\|_{0}^{0} .
$$

Since $\left\|\left(\operatorname{sgn} x\left(f-f_{n}\right)\right)^{\prime}\right\|_{0}^{-1} \leq\left\|f-f_{n}\right\|_{0}^{0}$, then $\widehat{\Psi} \Psi^{+} f \in \widetilde{H}_{0}^{-1}$ and (A.12) implies (A.10) for $f \in \widetilde{H}_{0}^{0}$. The lemma is proved.

By analogy with Lemma A.16, we obtain

Lemma A.17. Let $f \in \widetilde{H}_{0}^{-1}$. Then $\Psi \widehat{\Psi}^{+} f \in \widetilde{H}_{0}^{0}$

$$
\Psi \widehat{\Psi}^{+} f=\frac{1}{2} \operatorname{sgn} x\left(f *\left(\operatorname{sgn} x I_{0}(q x)-L_{0}(q x)\right)\right)=\operatorname{sgn} x \mathcal{F}_{\sigma \rightarrow x}^{-1}\left(\frac{(\mathcal{F} f)(\sigma)}{i \operatorname{sgn} \sigma \sqrt{\sigma^{2}+q^{2}}}\right), \quad f \in R(\widehat{\Psi}),
$$

and and $\left\|\Psi \widehat{\Psi}^{+}\right\| \leq \frac{\sqrt{1+q^{2}}}{q}$. Moreover, the operator $\Psi \widehat{\Psi}^{+}$can be continued on $\widetilde{H}_{0}^{-1}$ with the same norm by (A.13).

Lemma A.18. Let $f \in \widetilde{H}_{0}^{-1}$. Then $\operatorname{sgn} x f \in \widetilde{H}_{0}^{-1}$ and $\|\operatorname{sgn} x f\|_{0}^{-1} \leq 2\|f\|_{0}^{-1}$.

Proof. Let $\varphi \in \widetilde{H}_{0}^{1}$. Then $\|\operatorname{sgn} x \varphi\|_{0}^{1} \leq\|\operatorname{sgn} x \varphi\|_{0}^{0}+\left\|(\operatorname{sgn} x \varphi)^{\prime}\right\|_{0}^{0} \leq\|\varphi\|_{0}^{0}+\left\|\varphi^{\prime}\right\|_{0}^{0} \leq 2\|\varphi\|_{0}^{1}$. Hence, $\operatorname{sgn} x \varphi \in \widetilde{H}_{0}^{1}$. Since $\langle\operatorname{sgn} x f, \varphi\rangle=\langle f, \operatorname{sgn} x \varphi\rangle$, then $\operatorname{sgn} x f \in \widetilde{H}_{0}^{-1}$ and $\|\operatorname{sgn} x f\|_{0}^{-1} \leq 2\|f\|_{0}^{-1}$.

In Section 4 the continuation of $\widehat{\Psi} \Psi^{+}$on $\widetilde{H}_{0}^{0}$ has been denoted by $\Upsilon, D(\Upsilon)=\widetilde{H}_{0}^{0}$, and the continuation of $\Psi \widehat{\Psi}^{+}$on $\widetilde{H}_{0}^{-1}$ has been denoted by $\widehat{\Upsilon}, D(\widehat{\Upsilon})=\widetilde{H}_{0}^{-1}$. Lemmas A.16-A.18 yield

Corollary A.19. The operators $\Upsilon$, $\widehat{\Upsilon}$ are invertible, $\Upsilon^{-1} g=\mathcal{F}_{\sigma \rightarrow x}^{-1}\left(\frac{(\mathcal{F}(\operatorname{sgn} \xi g))(\sigma)}{i \operatorname{sgn} \sigma \sqrt{\sigma^{2}+q^{2}}}\right), D\left(\Upsilon^{-1}\right)=\widetilde{H}_{0}^{-1}, \widehat{\Upsilon}^{-1} g=$ $\mathcal{F}_{\sigma \rightarrow x}^{-1}\left(\operatorname{i} \operatorname{sgn} \sigma \sqrt{\sigma^{2}+q^{2}}(\mathcal{F}(\operatorname{sgn} \xi g))(\sigma)\right), D\left(\widehat{\Upsilon}^{-1}\right)=\widetilde{H}_{0}^{0}$, and $\left\|\Upsilon^{-1}\right\| \leq \frac{\sqrt{1+q^{2}}}{q},\left\|\widehat{\Upsilon}^{-1}\right\| \leq \sqrt{1+q^{2}}$.

Lemma A.20. Let $m=\overline{0, \infty}, \xi^{-2 m} \Phi \in H_{2 m}^{-2 m}, \operatorname{supp} \Phi \subset[0, q], \nu(\sigma, \xi)=\frac{2 \sigma}{\sigma^{2}+\xi^{2}}$ and $F(\sigma)=\langle\Phi, \nu(\sigma, \cdot)\rangle$. Then

$$
\|F\|_{0}^{0} \leq L_{m}\left\|\xi^{-2 m} \Phi\right\|_{2 m}^{-2 m}
$$

where $L_{m}>0$.

Proof. We have $\frac{1}{\sigma-\mathrm{i} \xi} \in H_{-2 m}^{2 m}$ (with respect to $\xi$ ), $\sigma \in \mathbb{R}$, and

$$
\begin{aligned}
F(\sigma) & =\frac{1}{\pi}\left\langle\Phi(\xi)+\Phi(-\xi), \frac{1}{\sigma-\mathrm{i} \xi}\right\rangle=\frac{1}{\pi}\left\langle\Phi_{1},\left(1+\xi^{2}\right)^{-m}\left(1+|D|^{2}\right)^{m} \frac{\xi^{2 m}}{\sigma-\mathrm{i} \xi}\right\rangle \\
& =\frac{2}{\pi} \int_{0}^{\infty} \Phi_{1}(\xi)\left(1+\xi^{2}\right)^{-m}\left(1+|D|^{2}\right)^{m} \frac{\xi^{2 m} \sigma}{\sigma^{2}+\xi^{2}} \mathrm{~d} \xi, \quad \sigma \in \mathbb{R},
\end{aligned}
$$


where $\Phi_{1}(\xi)=\left(1+\xi^{2}\right)^{m}\left(1+|D|^{2}\right)^{-m}\left(\xi^{-2 m}(\Phi(\xi)+\Phi(-\xi))\right) \in H_{0}^{0}$ and $\Phi_{1}$ is even. Then

$$
\begin{aligned}
\left(\|F\|_{0}^{0}\right)^{2}= & \frac{4}{\pi^{2}} \int_{0}^{\infty} \Phi_{1}(\xi) \int_{0}^{\infty} \overline{\Phi_{1}(\mu)} \\
& \times\left(1+\xi^{2}\right)^{-m}\left(1+\mu^{2}\right)^{-m}\left(1+\left|D_{\xi}\right|^{2}\right)^{m}\left(1+\left|D_{\mu}\right|^{2}\right)^{m} \xi^{2 m} \mu^{2 m} \int_{0}^{\infty} \frac{\sigma^{2} \mathrm{~d} \sigma}{\left(\sigma^{2}+\xi^{2}\right)\left(\sigma^{2}+\mu^{2}\right)} \mathrm{d} \mu \mathrm{d} \xi \\
= & \frac{4}{\pi^{2}} \int_{0}^{\infty} \Phi_{1}(\xi) \int_{0}^{\infty} \overline{\Phi_{1}(\mu)}\left(1+\xi^{2}\right)^{-m}\left(1+\mu^{2}\right)^{-m}\left(1+\left|D_{\xi}\right|^{2}\right)^{m}\left(1+\left|D_{\mu}\right|^{2}\right)^{m} \frac{\xi^{2 m} \mu^{2 m}}{\xi+\mu} \mathrm{d} \mu \mathrm{d} \xi
\end{aligned}
$$

For $\xi>0, \mu>0$ we have

$$
\begin{aligned}
& \left(1+\left|D_{\xi}\right|^{2}\right)^{m}\left(1+\left|D_{\mu}\right|^{2}\right)^{m} \frac{\xi^{2 m} \mu^{2 m}}{\xi+\mu}=\sum_{k=0}^{m} \sum_{p=0}^{m}\left(\begin{array}{c}
m \\
k
\end{array}\right)\left(\begin{array}{c}
m \\
p
\end{array}\right)(-1)^{k+p} \frac{\mathrm{d}^{2(k+p)}}{\mathrm{d} \xi^{2 k} \mathrm{~d} \mu^{2 p}} \frac{\xi^{2 m} \mu^{2 m}}{\xi+\mu} \\
= & \sum_{k=0}^{m} \sum_{p=0}^{m} \sum_{s=0}^{2 k} \sum_{l=0}^{2 p}\left(\begin{array}{c}
m \\
k
\end{array}\right)\left(\begin{array}{c}
m \\
p
\end{array}\right)\left(\begin{array}{c}
2 k \\
s
\end{array}\right)\left(\begin{array}{c}
2 p \\
l
\end{array}\right) \frac{((2 m) !)^{2}(s+l) !}{(2 k-s) !(2 p-l) !}(-1)^{k+p+s+l} \frac{\xi^{2(m-k)+s} \mu^{2(m-p)+l}}{(\xi+\mu)^{s+l+1}} .
\end{aligned}
$$

Therefore,

where

$$
\left|\left(1+\xi^{2}\right)^{-m}\left(1+\mu^{2}\right)^{-m}\left(1+\left|D_{\xi}\right|^{2}\right)^{m}\left(1+\left|D_{\mu}\right|^{2}\right)^{m} \frac{\xi^{2 m} \mu^{2 m}}{\xi+\mu}\right| \leq \frac{\pi^{2}\left(L_{m}\right)^{2}}{2(\xi+\mu)}, \quad \xi>0, \mu>0,
$$

$$
L_{m}=\frac{1}{\pi}\left(\sum_{k=0}^{m} \sum_{p=0}^{m} \sum_{s=0}^{2 k} \sum_{l=0}^{2 p}\left(\begin{array}{c}
m \\
k
\end{array}\right)\left(\begin{array}{c}
m \\
p
\end{array}\right)\left(\begin{array}{c}
2 k \\
s
\end{array}\right)\left(\begin{array}{c}
2 p \\
l
\end{array}\right) \frac{((2 m) !)^{2}(s+l) !}{(2 k-s) !(2 p-l) !}\right)^{1 / 2} \leq \frac{2}{\pi}(2 m) ! \sqrt{(4 m) !} 5^{m} .
$$

With regard to (A.15), we get

$$
\begin{aligned}
\left(\|F\|_{0}^{0}\right)^{2} & =2\left(L_{m}\right)^{2}\left\langle\left|\Phi_{1}(\xi) H(\xi)\right|,\left|\Phi_{1}(-\xi) H(-\xi)\right| * \frac{1}{\xi}\right\rangle \\
& \leq 2\left(L_{m}\right)^{2}\left(\left\|\Phi_{1}(\xi) H(\xi)\right\|_{0}^{0}\right)^{2}=\frac{1}{2}\left(L_{m}\right)^{2}\left(\left\|\Phi_{1}\right\|_{0}^{0}\right)^{2} \leq\left(L_{m}\right)^{2}\left(\left\|\xi^{-2 m} \Phi\right\|_{2 m}^{-2 m}\right)^{2} .
\end{aligned}
$$

That was to be proved.

\section{REFERENCES}

[1] M.I. Belishev and A.F. Vakulenko, On a control problem for the wave equation in $\mathbb{R}^{3}$. Zapiski Nauchnykh Seminarov POMI 332 (2006) 19-37 (in Russian); English translation: J. Math. Sci. 142 (2007) 2528-2539.

[2] I. Erdelyi, A generalized inverse for arbitrary operators between Hilbert spaces. Proc. Camb. Philos. Soc. 71 (1972) 43-50.

[3] L.V. Fardigola, On controllability problems for the wave equation on a half-plane. J. Math. Phys. Anal., Geom. 1 (2005) 93-115.

[4] L.V. Fardigola, Controllability problems for the string equation on a half-axis with a boundary control bounded by a hard constant. SIAM J. Control Optim. 47 (2008) 2179-2199.

[5] L.V. Fardigola, Neumann boundary control problem for the string equation on a half-axis. Dopovidi Natsionalnoi Akademii Nauk Ukrainy (2009) 36-41 (in Ukrainian). 
[6] L.V. Fardigola and K.S. Khalina, Controllability problems for the wave equation. Ukr. Mat. Zh. 59 (2007) 939-952 (in Ukrainian), English translation: Ukr. Math. J. 59 (2007) 1040-1058.

[7] S.G. Gindikin and L.R. Volevich, Distributions and convolution equations. Gordon and Breach Sci. Publ., Philadelphia (1992).

[8] M. Gugat, Optimal switching boundary control of a string to rest in finite time. ZAMM Angew. Math. Mech. 88 (2008) $283-305$.

[9] M. Gugat and G. Leugering, $L^{\infty}$-norm minimal control of the wave equation: on the weakness of the bang-bang principle. ESAIM: COCV 14 (2008) 254-283.

[10] M. Gugat, G. Leugering and G.M. Sklyar, $L^{p}$-optimal boundary control for the wave equation. SIAM J. Control Optim. 44 (2005) 49-74.

[11] V.A. Il'in and E.I. Moiseev, A boundary control at two ends by a process described by the telegraph equation. Dokl. Akad. Nauk, Ross. Akad. Nauk 394 (2004) 154-158 (in Russian); English translation: Dokl. Math. 69 (2004) 33-37.

[12] E.H. Moore, On the reciprocal of the general algebraic matrix. Bull. Amer. Math. Soc. 26 (1920) 394-395.

[13] R. Penrose, A generalized inverse for matrices. Proc. Camb. Philos. Soc. 51 (1955) 406-413.

[14] L. Schwartz, Théorie des distributions 1, 2. Hermann, Paris (1950-1951).

[15] G.M. Sklyar and L.V. Fardigola, The Markov power moment problem in problems of controllability and frequency extinguishing for the wave equation on a half-axis. J. Math. Anal. Appl. 276 (2002) 109-134.

[16] G.M. Sklyar and L.V. Fardigola, The Markov trigonometric moment problem in controllability problems for the wave equation on a half-axis. Matem. Fizika, Analiz, Geometriya 9 (2002) 233-242.

[17] J. Vancostenoble and E. Zuazua, Hardy inequalities, observability, and control for the wave and Schrödinder equations with singular potentials. SIAM J. Math. Anal. 41 (2009) 1508-1532. 